\title{
On developmental mental architectures
}

\author{
Juyang Weng* \\ Department of Computer Science and Engineering, Michigan State University, East Lansing, MI 48824, USA
}

Received 5 February 2005; received in revised form 15 June 2006; accepted 4 July 2006

Available online 21 January 2007

\begin{abstract}
This paper presents a computational theory of developmental mental architectures for artificial and natural systems, motivated by neuroscience. The work is an attempt to approximately model biological mental architectures using mathematical tools. Six types of architecture are presented, beginning with the observation-driven Markov decision process as Type-1. From Type-1 to Type-6, the architecture progressively becomes more complete toward the necessary functions of autonomous mental development. Properties of each type are presented. Experiments are discussed with emphasis on their architectures.
\end{abstract}

(C) 2007 Published by Elsevier B.V.

Keywords: Mental architecture; Agent architecture; Computational neural science; Cognitive development; Autonomous mental development; Developmental robots; Learning types; Developmental vision; Speech recognition; Language acquisition; Thinking; Reasoning; Autonomous planning

\section{Introduction}

This paper deals with the architecture level of a biologically inspired system that is capable of autonomous mental development (AMD). By AMD, we mean an agent, natural or artificial, improves its mental (perceptual, cognitive, behavioral and motivational) capabilities through autonomous interactions with its environments. Mental architecture is a challenging and important subject, but there have been relatively few systematic (agent-wise) investigations. Further, the working of the mind is hard to understand without understanding AMD. A mathematically rigorous investigation of mental architecture is necessary in order to achieve this understanding.

A system that senses and acts is called an agent. In the machine learning community, the agent architecture has been a very important and extensively studied subject. Supervised and reinforcement learning, based on the Markov decision Process (MDP) architecture (single- or multi-level), enables a robot (an embodied agent) to learn autonomously while the environment (including humans) provides labels for supervised learning [28] or rewards for reinforcement learning $[14,34]$. The model is for a general

\footnotetext{
*Tel.:/fax: + 15173534388 .

E-mail address: weng@cse.msu.edu.
}

purpose, in that given an appropriate task, a human programmer can establish the correspondence between every state (node) and the designed task-specific concept, before the initialization and learning of MDP take place. However, the MDP architecture, as explained in the following sections, has fundamental limitations that prevent it from being effective for the developmental robots described in [47]. A major limitation of the MDP model is its non-developmental nature, in the sense that the meanings of the states (or nodes) are fixed (manually designed instead of observation driven).

Additional general-purpose architectures have been proposed by researchers in artificial intelligence and psychology. Major remarkable ones include Soar proposed by Laird et al. [17], ACT-R by Anderson [3], the architecture by Albus [1], and the subsumption architecture by Brooks [6]. Soar and ACT-R incorporated many useful concepts that are necessary for human intelligence. Albus' architecture outline was motivated by neural architecture and gives a temporal view of a complex mental architecture. The subsumption architecture is a biologically motivated architecture component, motivated by motor cortex. It provides a way to decompose the architecture according to behaviors.

The architecture models discussed above do not directly address perception, such as vision and audition. Neither do 
the models that aim to model human performance, e.g., error rate (see, e.g., EPIC by Meyer and Kieras [20,21]) as opposed to actual mental capabilities. A common limitation of these architectures is in the use of symbolic representation in internal representation. Inspired by the biological brain (e.g., cortical representation) and its development, no task-specific symbolic representation will be used in the proposed developmental architectures. Distributed (i.e., vector), receptor- and effector-derived numeric representation is used through the proposed series of mental architectures.

Perceptual architecture is still an unsolved, important, yet very challenging problem. Neisser [23] pointed out that any model of vision that is based on spatial computational parallelism alone is doomed to failure. He proposed a twostage visual process which consists of a pre-attentive phase followed by an attentive phase. Feldman and Ballard [9] proposed a "100-step rule:" A biologically plausible algorithm for immediate vision (one that does not involve slower deliberate thinking) can require no more than 100 steps. John Tsotsos' study [36] on the complexity of immediate vision proposed a coarse architecture for a biologically motivated general purpose vision system (for immediate vision).

All the above architectures are nondevelopmental in the sense that the information processor is not generated through real-time interactions with the environment. In other words, a developmental architecture requires not only a specification of processors and their interconnections, but also their online, incremental, automatic generation from real-time experience.

Recently, there has been an onset of efforts on computational studies of AMD (e.g., the Workshop on Development and Learning [48], the series of International Conferences on Development and Learning, and the International Workshops on Epigenetic Robotics). Although many of the published studies have not reached fully autonomous incremental online development, these studies are very important for understanding AMD by higher animals and the realization of artificial AMD by robots. Darwin V robot [2], for example, had plastic association between feature extractors and motors, although other parts are not plastic. A reason for this status quo is the lack of studies on developmental mental architecture. This paper deals with this important issue.

The paper presents a series of architectures, from the simplest Type 1 to the full Type 6 where every module is plastic (developmental). The paper does not describe our developmental algorithms that use such architectures, but cites the related publications that specify the full detail for every module. The paper also discusses some of our experimental systems that use the architecture models described here. The computational models proposed here are inspired by networked neurons, cortical regions and their development. The correspondence of the architecture components with brain regions is discussed, but an exact provable correspondence is not possible at the current state-of-the-art.
It is difficult to be both biologically inspired and mathematically grounded, which the work presented here strives to achieve. Like the architecture of MDP [28,14,34], the presentation of the series of mental architectures is in mathematical terms, which is necessary for a clear and rigorous understanding of the related concepts. The brain operates quantitatively (e.g., the cortex is a function); and mathematics is a powerful tool to model quantitative relationships. It is also necessary to derive the mathematical properties of the corresponding architecture so that the power and the limitation of the architecture are better understood.

The work reported here is a theoretical, computational, and experimental step toward the goal of understanding mental architecture. This paper does not intend to solve all the problems and answer all the questions about this subject. However, it is for the first time, as far as the author knows, a systematic presentation of a series of biologically inspired mental architectures in rigorous mathematical terms. Many of the architecture concepts and properties presented here are new (e.g., Types 3 to 6).

In the following sections, I first introduce a series of architectures, from simple to complex, along with the associated properties. Then, in Section 9, I propose a series of new modes of learning that are necessary for understanding various modes of development as well as their differences with traditional machine learning modes. In Section 10, the presented architectures are related to our experimental systems.

\section{Type-1: Observation-driven MDP (ODMDP)}

A major drawback of the traditional mental architectures is that they do not enable the agent to sense the conscious part of its mental activities. In 2002, I introduced the concept of self-aware self-effecting (SASE) agent $[42,43]$, but the architecture that is required for SASE agents has not been fully presented in rigorous mathematical terms and the following fills the void.

\subsection{Internal and external environments}

In mathematical terms, the brain (or central nervous system) of an agent consists of all the signal processors between sensory inputs (receptors) and the effector outputs (motors). First, we need to formally define the concept of internal environment.

Definition 1. The internal environment of an agent is the brain (or "the central nervous system" (CNS)) of the agent. The external environment consists of all the remaining parts of the world, including the agent's own body (excluding the brain).

These concepts are biologically based and are extended to developmental robotics. The term "internal" refers to the brain only (since we deal with mental architectures). The physical body of the agent is a part of the external 
world. This raises a natural question for the sensors and effectors: Are sensors and effectors internal or external? In biology, they belong to the peripheral nervous system and the body, and therefore, are considered external. For example, the camera of the robot and the eyes of a human are external to the brain. The video signals from the camera are transmitted from the camera (external) to the central nervous system (internal). Likewise, the receptors in the retina (external) generate nervous signals that are sent to the lateral geniculate nucleus (LGN) and the primary cortex in the human central nervous system.

Definition 2. An external sensor $S_{e}$ and an internal sensor $S_{i}$ are sensors that sense the external and internal environments, respectively. An external effector $E_{e}$ and an internal effector $E_{i}$ are effectors that act on the external and internal environments, respectively.

The role of a sensor is to transform one form of physical energy (e.g., optical) into another (nervous spikes). This results in an interesting situation in the biological nervous system: Although some nervous projections serve the role of internal sensors, there are no physical receptors to help to identify these internal projections. For example, the internal attention selections from later processing back into early processing (e.g., the primary cortex) are sensed as action inputs (as a self-aware attention selection action), but there are no physical receptors along these projections, since the form of signal is already in the desired form (nervous spikes). The concept of (virtual) internal sensors introduced here could be useful for understanding the circuit basis of self-awareness.

\subsection{Interactive agents}

With the knowledge of internal and external environments, we are ready to introduce the concept of agent. Fig. 1 illustrates a multi-sensor multi-effector model of agent. An agent $A(t)$ operates at equally spaced discrete time instances $t=0,1, \ldots$. The time unit is called (discrete) mental cycle. A typical mental cycle lasts about $33 \mathrm{~ms}$ for a refreshing rate of $30 \mathrm{~Hz}$. In other words, the refreshing rate is considered as the digital sampling rate of the nervous signals represented by the faster neuronal spikes.

We assume that an image is produced at each discrete time instant by the sensor, independent of the sensing modality (visual, auditory, touch, etc.). In Fig. 1, without loss of generality, we assume that the agent has two external sensors and two external effectors. Each external sensor $S_{e i}, i=1,2$, senses a random multi-dimensional sensory frame $x_{e}(t)=\left(x_{e 1}(t), x_{e 2}(t)\right)$ at each time instant $t$ and the sensed signal is fed into the agent. Each external effector $E_{e i}, i=1,2$, receives from the agent an effector frame $a_{e}(t)=\left(a_{e 1}(t), a_{e 2}(t)\right)$ at each time instant $t$. Note that we change a variable of a vector, which is typical for a continuous variable, to its subscript (e.g., change $x(t)$ to $x_{t}$ ) when it is necessary to indicate its sampled, discrete version.

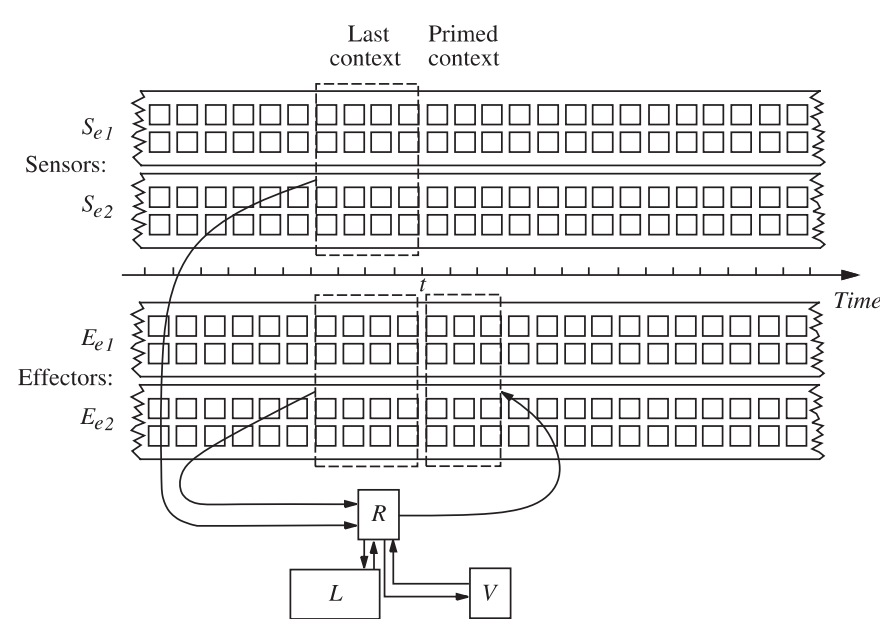

Fig. 1. The Type-1 architecture of a multi-sensor multi-effector agent: observation-driven Markov decision process. In the temporal sensory and effector streams, each square denotes a receptor (e.g., pixel) or motor and vertically aligned squares form a time frame. The Type- 1 architecture takes the entire image frame without applying any mask. The block marked with $L$ is a set of context states (prototypes), which are clusters of all observed context vectors $l(t)$.

\subsection{Markov decision process}

As we stated before, we need to address an agent's mental architecture based on rigorous mathematical formulation which allows us to further study properties and limitation. We start with the MDP, which is a framework originated from mathematics and statistics and has been studied and used widely in the machine learning community $[27,14,34]$.

Definition $3(M D P)$. The MDP is as follows. Suppose $S=$ $\{1,2, \ldots, n\}$ is a set of $n$ predefined symbolic states that is used to model a part of the world. The state $s_{t}$ at time $t$ is a random variable taking one of the values in $S$. Its prior probability distribution is $P\left(s_{0}\right)$. The action $a_{t}$ is the action of the agent at time $t$. Let $H_{t}$ be the random history from time $t=0$ up to time $t-1$ :

$H_{t}=\left\{s_{t-1}, s_{t-2}, \ldots, s_{0}, a_{t-1}, a_{t-2}, \ldots, a_{0}\right\}$.

If its conditional state transitional probability $P\left(s_{t} \mid H_{t}\right)$ satisfies

$P\left(s_{t} \mid H_{t}\right)=P\left(s_{t} \mid l_{t}\right)$,

where $l_{t}$ is the short last $k$ frames of the history

$l_{t}=\left\{s_{t-1}, s_{t-2}, \ldots, s_{t-k}, a_{t-1}, a_{t-2}, \ldots, a_{t-k}\right\}$,

we call it the $k$ th order MDP $[27,14,34]$.

Many well-known cognitive architectures that are based on symbolic states are compatible with MDP, except that the probability distribution degenerates into a single-point distribution, with no modeling of uncertainty. They include the schema mechanism, the production systems and their extensions such as Soar, ACT-R, and Gary Drescher's architecture which simulates some aspects of Piaget's constructivist theory. Of course, this compatibility does 
not mean that MDP architecture includes many other features of all those systems.

In addition to missing many key architecture components to be introduced later in the paper, the first limitation of MDP is that it assumes the state is directly observable. In many applications, the state of the world is not directly observable by the agent, or is partially observable (with noise).

Definition 4 (Partially observable MDP). If the state $s_{t}$ of the world is not totally observable to the agent and, instead, there is an observation $x_{t}$ at time $t$ that depends on the state $s_{t}$ by an observation probability $P\left(x_{t} \mid s_{t}\right)$, the process is called partially observable MDP or POMDP $[14,34]$ (or HMM [28]).

\subsection{Observation-driven Markov decision process}

MDP and POMDP as formulated above has been widely used to model a process in the external environment. However, they are not suited for modeling the mental process in the internal environment (CNS) and especially not suited for modeling developmental architecture. Therefore, we consider the following ODMDP.

Definition 5 (Type-1). Let $x_{t} \in \mathcal{X}$ and $p_{t} \in \mathcal{P}$ be the observations and outcome covariates (i.e., random vectors) at time $t$, respectively. Let $H_{t}$ be the random vector of the entire history:

$H_{t}=\left\{x_{t}, x_{t-1}, \ldots, x_{0}, p_{t-1}, \ldots, p_{0}\right\}$.

If its state transitional probability $P\left(s_{t} \mid H_{t}\right)$ satisfies

$P\left(p_{t} \mid H_{t}\right)=P\left(p_{t} \mid l_{t}\right)$,

where $l_{k}$ is the last $k$ observations:

$l_{t}=\left\{x_{t}, x_{t-1}, \ldots, x_{t-k}, p_{t-1}, \ldots, p_{t-k}\right\}$

as shown in Fig. 1, we call the process as the $k$ th order ODMDP [7]. We define the Type-1 mental architecture as a $k$ th order ODMDP.

Let us consider why ODMDP is suited for a developmental architecture. In the developmental ODMDP, the random observations in $l_{t}$ across time $t=0,1, \ldots, t$ are the source from which the agent automatically generates states in the form of clusters (corresponding to neurons with their synaptic weights) $l \in \mathcal{L}$, where $\mathcal{L}$ consists of all possible observations of the last contexts $\mathcal{L}=\left\{l_{t} \mid 0 \leqslant t\right\}$. The predicted consequence $p_{t}$ consists of predicted action $a_{t}$ (e.g., motor signals) and the predicted value $v_{t}, p_{t}=\left(a_{t}, v_{t}\right)$.

The following are the major differences between a POMDP (or HMM) and an ODMDP:

1. The POMDP is world-centered, where each state corresponds to a modeled object or event of the world (e.g., a corner). The ODMDP is mind-centered (from sensors), where each state corresponds to an observation from the environment (e.g., a view of a corner with other background objects).
2. The states $s_{t}$ of POMDP are hand-specified but the states of ODMDP can be automatically generated (developed) on-the-fly. With the POMDP, the meaning of each state must be specified so that the initial estimates of the three probability distributions $\left(P\left(s_{0}\right)\right.$, $P\left(s_{t} \mid s_{t-1}\right)$ and $\left.P\left(x_{t} \mid s_{t}\right)\right)$, can be provided.

3. In the POMDP, there are two layers of probability: the state transition probability $P\left(s_{t} \mid x_{t}, s_{t-1}\right)$ and the state observation probability $P\left(x_{t} \mid s_{t}\right)$, while the ODMDP has only one layer of probability: $P\left(p_{t} \mid l_{t}\right)$, making a more efficient learning algorithm possible.

\subsection{Type-1: An observation-driven regressor with values}

It is not difficult to accept that each cerebral cortex is a multidimensional mapping. However, what domain it maps from and what codomain it maps to have not been clearly understood. In other words, what does each cortical area do? Each cerebral cortex realizes a regressor $R$. Mathematically, a regressor $R$ is a mapping between the space of input $\mathcal{X}$ (the space of receptors or the output space of feature detectors) and the space of output $\mathcal{Y}$ (the space of primed context), denoted as $R: \mathcal{X} \mapsto \mathcal{Y}$, so that for each input $X \in \mathcal{X}$, there is an output $Y=R(X)$. It is a great challenge to incrementally generate $R$ from real-time experience (i.e., cortical development). We implemented the regressor $R$ using the incremental hierarchical discriminant regression (IHDR) [45,44]. Given any observed (last) context $l(t)$, the regressor $R$ produces multiple consequences (primed contexts) $p_{1}(t), \ldots, p_{k}(t)$ that have a high probability:

$\left\{p_{1}(t), \ldots, p_{k}(t)\right\}=R(l(t))$.

The set of primed contexts is constrained by innate mechanisms (e.g., inborn reflexes) and experience (e.g., practice and exploration). Thus, the regressor $R$ is a mapping from the space of the last context $\mathcal{L}$ to the power set $^{1}$ of $\mathcal{P}$, denoted as $2^{\mathcal{P}}$ :

$R: \mathcal{L} \mapsto 2^{\mathcal{P}}$.

In other words, given any pattern of input (context) to $R$, there are a multiple primed contexts as output. For example, upon seeing a Y-junction, your brain pops up multiple actions: left turn and right turn.

In both biological and robotic CNS, $R$ is developed incrementally and adaptively through, and is greatly shaped by, real-time prenatal and postnatal experience, constrained by the innate genetic information (developmental mechanisms).

At the Y-junction, which turn is better? This is the role of the value system. The value system $V(t)$ (also called motivational system in neuroscience [15]) selects a desirable context from multiple primed ones:

$$
V(R(l(t)))=V\left(\left\{p_{1}(t), p_{2}(t), \ldots, p_{k}(t)\right\}\right)=p_{i}(t),
$$

\footnotetext{
${ }^{1}$ The power set of $A$ is the set that consists of all possible subsets of $A$.
} 
where $1 \leqslant i \leqslant k$ and $k$ varies according to experience. The value function selects the best consequence $p_{i}(t)$ that has the best value $v_{i}(t)$ in $p_{i}(t)=\left(a_{i}(t), v_{i}(t)\right): i=\arg \max \left\{v_{1}(t)\right.$, $\left.v_{2}(t), \ldots, v_{k}(t)\right\}$. These values are constrained by genetic information and are accumulated from corresponding experiences in the past, such as inborn reflexes, inborn preferences (e.g., pain and pleasure), adaptive values (e.g., novelties in the process of sensitization and habituation), learned values (classical conditioning and instrumental conditioning) and social values (e.g., liked by parents or peers).

We applied the real-time $Q$-learning algorithm [41] to estimate the value $v_{i}$ of each consequence $p_{i}(t)$, $i=1,2, \ldots, k$, by handling delayed rewards [43] (e.g., a reward is typically delivered after the action is delivered). Therefore, the value system $V$ is a mapping from the power set of $\mathcal{P}$ to the space of $\mathcal{P}$ :

$V: 2^{\mathcal{P}} \mapsto \mathcal{P}$,

meaning that it selects a single primed context from multiple contexts. Combining Eq. (2) with Eq. (4), we see that the regressor $R$ followed by the value system $V$ accomplish the following combined mapping from the space of last context $\mathcal{L}$ to the space of primed context $\mathcal{P}$ :

$\mathcal{L} \stackrel{R}{\mapsto} 2^{\mathcal{P}} \stackrel{V}{\mapsto} \mathcal{P}$.

Therefore, although the brain appears to always take a single action, it evaluates multiple choices on the fly in each mental cycle (e.g., 100-200 ms). This relatively fast, mentalcycle based mapping considers multiple primed contexts in $\mathcal{P}$. It participates in, but is not necessarily the same as, the conscious, autonomous thinking process that covers a significantly longer time scale.

\section{Type-2: Observation-driven selective MDP}

The Type-1 mental architecture is sensory nonselective in the sense that it is not able to actively select a subpart of relevant information from the sensory frame (i.e., intramodal attention) or to attend a particular modality but not the other (i.e., inter-modal attention). Many of the well known architectures are nonselective by themselves, including POMDP, HMM, Soar, and ACT-R. The need of attention selection as an integral part of mental architecture has been largely ignored in the machine intelligence community, except the computer vision community.

\subsection{Selective}

In the computer vision community, attention selection has been dealt with in ad hoc, task-specific ways (see e.g., $[5,29])$. John Tsotsos' study on the complexity of immediate vision $[35,36]$ and Zhang and Weng's general-purpose attention selection mechanisms (SHM + HDR) [51] are two notable exceptions in the computer vision community. In neuroscience and psychology, the role of attention selection in visual processing is well recognized [38,24,30], but detailed neural computational architectures that integrate attention selection in early and later processing are still lacking.

Given a $d$-dimensional input vector $x$, the attention can be modeled by an attention mask $m$, where $m$ is a $d$-dimensional vector whose elements are either 0 or 1 . Suppose that the input vector is $x=\left(x_{1}, x_{2}\right)$ and the mask is $m=\left(m_{1}, m_{2}\right)$. Then the corresponding attended input vector is $x^{\prime}=x \otimes m=\left(x_{1} m_{1}, x_{2} m_{2}\right)$, where $\otimes$ denotes vector pointwise product. Not all the masks are admissible. For example, the set of admissible masks consists of circles with different radiuses $\rho$ at different center positions $\left(r_{0}, c_{0}\right)$ of the image frame. Then, the attention selection effector has three degrees of freedom: $\left(r_{0}, c_{0}, \rho\right)$.

Definition 6 (Type-2). The Type-2 mental architecture is a Type-1 architecture, with the addition of an attention selector, more generally called sensory mapping,

$T: \mathcal{Y} \times \mathcal{A}_{i} \mapsto \mathcal{L}$,

as shown in Fig. 2, where $\mathcal{Y}$ is the space of all possible preattention contexts $\mathcal{Y}=\{l(t) \mid 0 \leqslant t\}, \mathcal{A}_{i}$ is the space of all possible attention selections for $T$, and $\mathcal{L}$ is the space of attention-masked, last contexts.

For example, the set $\mathcal{A}_{i}$ consists of sampled circles of attention centered at different retinal positions $(x, y)$ with radius $\rho$. The circles are sampled because the number of receptors in the retina is finite. Thus, the control of $T$ has only three parameters $(x, y, \rho)$.

It is known that there are extensive projections from later cortical areas (e.g., the prefrontal and frontal cortices) to early cortical areas (e.g., V1, V2, V3, V4, IT). The early

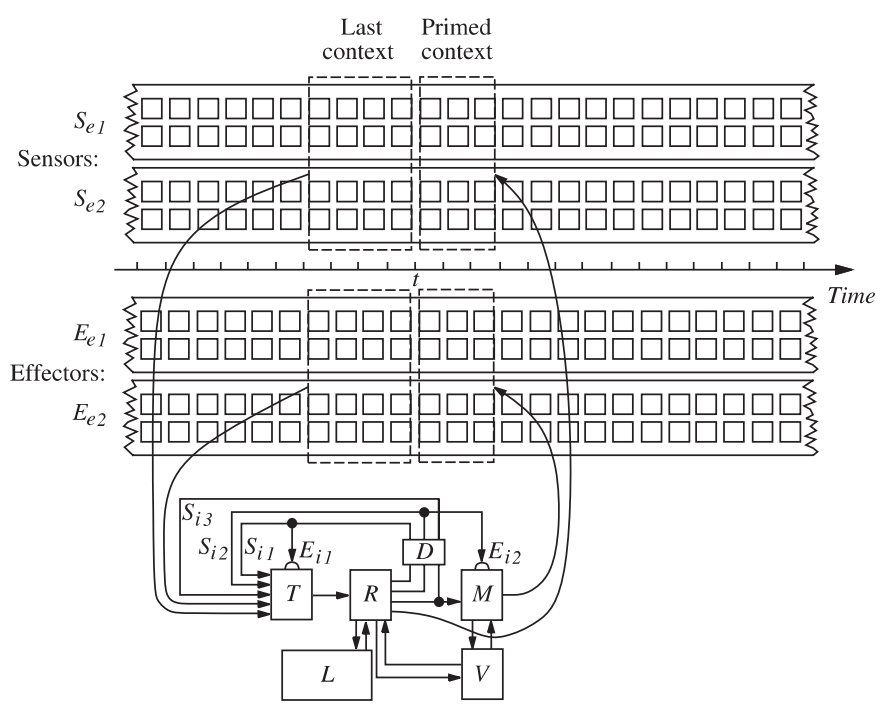

Fig. 2. Progressive additions of architecture components from Type-2 to Type-5. Type-2: adding attention selector $T$ and its (internal) control input $E_{i 1}$. Type-3: Adding motor mapping $M$ and its (internal) control $E_{i 2}$. Type-4: Adding internal controls $S_{i 1}$ and $S_{i 2}$ and the primed sensation $S_{i 3}$ to the entry port of perception $T$. The block marked with $D$ is a delay module, which introduces a unit-time delay for the corresponding vector. Type-5: Developmental $T, R, M$ and $V$. 
cortical areas are likely the biological basis for the attention selector $T$ while the later processing cortical areas generate attention selection signals in $\mathcal{A}_{i}$.

Luria [18] divided the cortex into two functional units. The posterior portion of the neocortex (parietal, temporal, and occipital lobes) is the sensory unit and the anterior cortex (frontal lobe) is the motor unit. If the architecture is plotted in such a way that sensory input is from the left and motor output is toward the right, Luria's division can be called the within-sensorimotor hierarchy. It approximately corresponds to: (1) primary sensory areas $T$; (2) association areas $R(L), V$; and (3) motor areas $M$. Therefore, mathematically, we call $T$ the sensory mapping, $R$ the associative (or cognitive) mapping, and $M$ the motor mapping.

In the Type- 2 architecture, $T$ corresponds to the sensory components that are single modal (e.g., visual). The attention selector $T$ is spatial and temporal specific (e.g., retinotopic) through which the brain can actively select responses from specific areas. For vision, $T$ may correspond to the retina, LGN and V1. The regressor $R$ corresponds to Luria's visual secondary I (Brodmann's areas 18 and 19), visual secondary II (areas 20, 21, 37) and tertiary sensory (areas 38, 39, 40). Based on the schematic diagram of Kolb [16] (p. 195), Fig. 3 illustrates the correspondence between the cortical areas and the components of the introduced architecture, using vision as an example. However, this type of architecture is not unique for vision. Almost identical architectures exist for auditory and somatosensory pathways, and the same is probably true for taste and olfaction [16] (p. 196).

Traditionally, in many symbolic systems such as Soar and ACT-R, the production rules are in the form of "if-then" pairs. The conditions following "if" are in a hand-predesigned symbolic form. These architectures do not model attention model $T$ as the executor of attention selector $T$. Lack of the attention selector $T$ is a fundamental limit that prevented these systems from realizing sensor-based autonomous learning. Therefore, those traditional architectures are not suited (or not designed) for dealing with real, non-text sensors directly. Further, lack of attention selector $T$ prevents Soar and ACT-R from learning to autonomously select different sources, a capability critical for mental development.

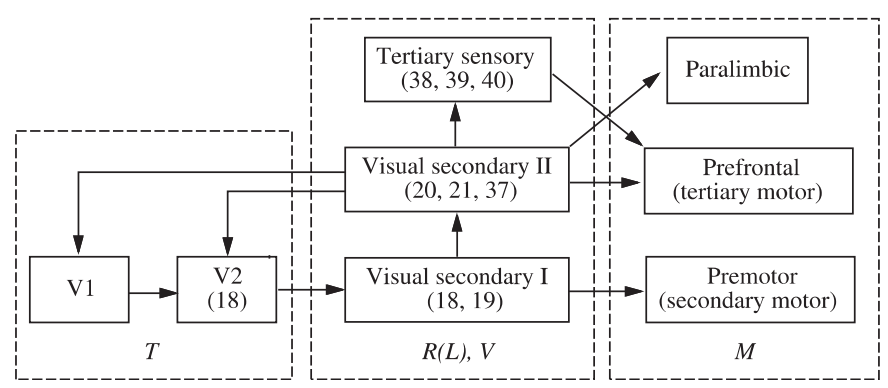

Fig. 3. Schematic illustration of the within-sensorimotor hierarchy, with the visual sensorimotor pathway as an example and the correspondence with the cortical areas.

\subsection{Higher architecture}

In order to investigate the properties of different architectures, we define a concept called higher architecture.

Definition 7 (Higher architecture). Given a set $D$ of tasks, we say that a developmental architecture $A_{2}$ is higher than another developmental architecture $A_{1}$, if given the same teaching environment $E$, the architecture $A_{2}$ requires statistically fewer teaching examples than $A_{1}$, expected over the environment $E$ and over the tasks in $D$, to reach the same performance.

As a convention, we regard environment as part of the specification of a task. For example, a task is more challenging if the environment of the task execution is uncontrolled. The performance metrics are important. Sometimes in real world AMD in humans and robots, slower building of networks may yield higher abstractions (e.g., in language acquisition) [8].

Theorem 1 (Existence of higher architecture). There is at least one class of tasks and the associated teaching environment $E$ in which the Type-2 architecture is higher than the Type-1 architecture.

Due to the limit of space, here only a sketch of proof is provided. Construct a class of tasks whose goal is to classify sensory information in a given set. The Type-2 architecture enables the teacher to teach the agent to pay attention to $S_{e 1}$, but the Type-1 architecture cannot.

Biologically, a lower mental architecture could be possessed by lower organisms such as insects, and a higher architecture could be that of higher animals such as mammals. However, at this point, the Type-1 and Type-2 distinctions have not been established for biological species. Architecture differences among biological organisms could be a subject of future study. However, it cannot be ruled out that even insects still possess a Type-2 architecture and the difference between insects and mammals is the sophistication of the attention selector $T$.

Clearly, a major rule of attention selection is to generalize better in new settings. E.g., in order to understand new settings where a familiar human face appears in new backgrounds, the agent architecture must enable the agent to pay attention to the human face and the background components separately. The higher architecture enables better generalization, but requires additional skills (e.g., attention selection). A higher architecture typically requires more sophisticated learning.

\section{Type-3: observation-driven selective rehearsable MDP}

The Type-2 architecture does not have a motor mapping $M$. Therefore, it cannot autonomously rehearse an action sequence to evaluate its consequences without actually carrying out the action sequence. 


\subsection{Rehearsable}

The desired rehearsal is autonomous in that there are no pre-defined program segments that specify when and how to rehearse.

Definition 8 (Type-3). The Type-3 mental architecture is a Type- 2 mental architecture, with the addition of an action releaser $M$ :

$M: \mathcal{P} \times \mathcal{A}_{i} \mapsto \mathcal{P}$,

as shown in Fig. 2, where $\mathcal{P}$ is the space of all possible predicted consequences, $\mathcal{A}_{i}$ is the space of all possible attention selections for $M$.

The action releaser $M$ is a special case of the prefrontal, pre-motor and motor cortices as illustrated in Fig. 3, which has other functions such as generating representation for frequently practiced action sequences (e.g., using the principal component analysis PCA or independent component analysis ICA) so that smooth action sequences can be generated.

\subsection{Why mental rehearsal}

With a traditional MDP with hand-designed states, it is possible to compute all the possible next states and perform planning. The $Q$-learning method [41] uses the estimated action value $Q(s, a)$ of action $a$ at state $s$ to select the best action $a^{*}=\max _{a \in A} Q(s, a)$, from the set $A$ of all the possible actions. This best next action $a^{*}$ maximizes the expected rewards in the future. The same is true for other SARSA models with exploration. This kind of approach has two fundamental problems. First, the value system is rigid. No matter what value model is used (finite horizon, time discount model, exploration, etc.), the agent cannot autonomously change the way the value is determined $[14,34]$. For example, if the time discount model is used, the agent is short-sighted. It prefers small rewards in the near future to a faraway but important reward. The system is not able to mentally rehearse actions (e.g., by talking to itself). Instead, it strictly experiences the event and receives rewards. After the robot has run into a river, for example, it is dead already and no reward is useful at that time.

However, although the Type- 3 architecture allows the agent to learn rehearsal, it cannot learn to rehearse through autonomous internal thinking because it is not aware of internal actions. Can we design an architecture that enables the robot to autonomously "consider" and "plan" a significant amount of time ahead before it releases the action? The next type makes it possible.

\section{Type-4: Observation-driven SASE MDP}

The type- 4 architecture is SASE. The term "self" here means the brain, instead of the body of the agent.

\subsection{Awareness}

Definition 9 (Awareness). The awareness of a task $b$ in an (internal and external) environment $E$ by an agent $A$ is the capability of the agent to (1) sense various context states $s$ of task $b$ from $E$ and (2) recall the predicted multiple contexts (primed contexts) $p=R(s)$ using the regressor $R$.

The awareness here is not consciousness (e.g., metacognition). It is the architecture necessity that may potentially enable consciousness after development. But by itself alone, it not able to realize metacognition.

By this definition, the agent must use its sensors, the entry point of its sensory architecture (the input of $T$ ), to sense the contexts. In the above definition for awareness, we consider a particular $b$ and an environment $E$. This is because any awareness has a scope. A person who is aware of the boiling temperature of water in a domain (e.g., in a normal environment) may not necessarily be aware of the boiling temperature of water in another domain (e.g., lower in a low pressure environment). With the above definition, we are ready to address the issue of SASE.

Theorem 2 (Necessary conditions of self-awareness). Suppose an agent is aware of its mental activities (sensations and actions) about a task $b$ in an environment $E$. Then the following must be true: (1) It senses such mental activities using its sensors. (2) It feeds the sensed signal into its perception entry port $T$ just like that for external sensors.

Proof. Point (1) is true because, according to the definition for the awareness of an object, the agent must sense the object using its sensors. Point (2) is true because the status of the object must be sensed and fed into the entry port for sensors for proper perception and recall of the primed contexts.

Based on the last two theorems, let us examine the issue of self-awareness more closely. If an agent runs a learning algorithm (e.g., the $Q$-learning algorithm) but it does not sense the voluntary decision process using the sensors that are linked to its entry point for sensors, the agent is not aware of its own algorithm. For the same reason, humans do not sense the way their primary cortex works and, therefore, normally they are not aware of their own earlier visual processing. However, the voluntary part of the mental decision process does require a conscious, willful decision. Therefore, the point is not that the brain has internal input and output functions within the brain (all models with multiple layers of hidden units already make this assumptions). The point is that these internally sensed signals must be fed into the entry port $T$ for perception if the system needs to be aware of such signals.

\subsection{Self-aware and self-effecting}

The traditional model of an agent has a fundamental flaw. The model is for an agent that only perceives and acts 
on the external environment. It does not sense its internal "brain's" activities. In other words, its internal decision process is neither a target of its own cognition nor a subject for the agent to explain when the agent is sufficiently mature. For example, Soar and ACT-R architectures do not enable the corresponding system to sense the internal reasoning activities.

The human brain allows the thinker to sense what he is thinking about without performing an overt action. For example, visual attention is an SASE internal action (see, e.g., Kandel et al. [15, pp. 396-403]). An action for attention selection generated by the later motor area is projected back to the sensory processing area to suppress visual areas that are not of interest. The value of an event (e.g., a dangerous setting) generated by the value area is perceived (e.g., What will happen? Do I like what will happen?) by the sensory area and is used by the decision process. Motivated by neuroscience, the mathematical model of the SASE agent, shown in Fig. 4, is defined as follows.

Definition 10. An SASE agent has internal sensors $S_{i}$ and internal effectors $E_{i}$ for this internal (brain) environment, in addition to its external sensors $S_{e}$ and external effectors $E_{e}$ for its external environment (outside brain). The regressor $R$ takes signals from $S_{i}$ and $S_{e}$ and generates internal and external actions for $E_{i}$ and $E_{e}$, respectively.

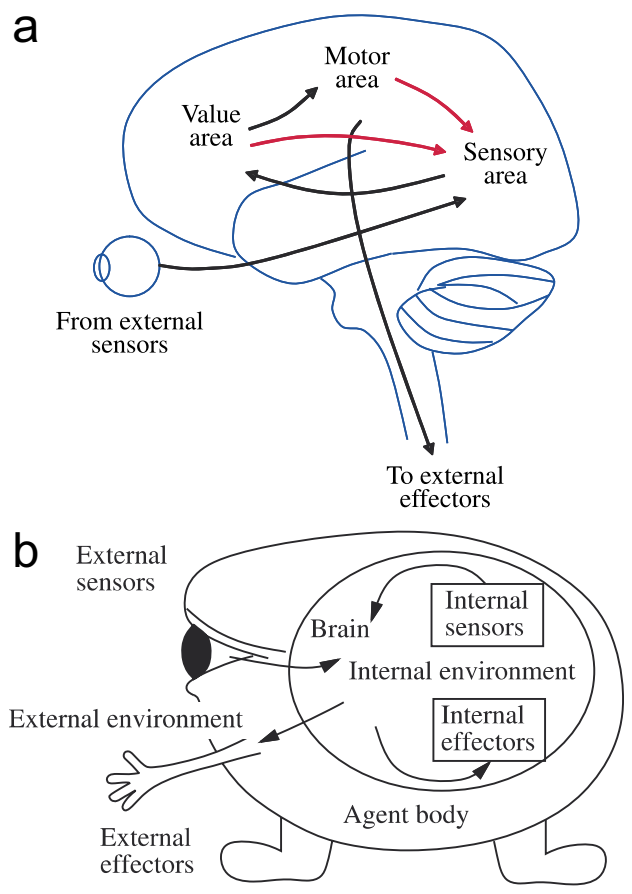

Fig. 4. (a) The brain is a SASE Agent. It interacts with not only the external environment but also its own internal (brain) environment: the representation of the brain itself. The afferent (out-going) projections from the motor area and the value area correspond to equivalent internal sensors. The efferent (receiving) projections from the motor area to the sensory area correspond to equivalent internal effectors. (b) The equivalent internal sensors and internal effectors of a Self-Aware SelfEffecting (SASE) agent.
A SASE agent perceives and interacts with not only the external environment but also the conscious part of its own internal (brain) environment: the representation of the brain itself.

The major design principles for a SASE agent include:

1. A SASE agent must have a sensor for each of its voluntary external effectors, so that it can sense what each effector is doing. For example, the muscle spindles sense the tension of human muscles to tell the position of the arms.

2. A SASE agent must have internal effectors and internal sensors for its voluntary internal effectors. For example, it needs a set of internal attention effectors to select the most relevant part of sensory information for later processing, which eventually leads to voluntary actions for attention selection.

3. A SASE agent needs pre-motor areas, where it stores information about the control of the effectors, but the signal in the pre-motor area is not sent to the effectors unless an action release signal is issued. The effector signals in the pre-motor areas are also sensed by internal sensors so that the robot can "talk to itself" internally.

It is important to note that not all the internal brain representations are sensed by the human brain itself. Early processing actions are typically not sensed.

Definition 11 (Type-4). The Type-4 mental architecture is a Type-3 mental architecture, but additionally, the internal voluntary decision is sensed by the internal sensors $S_{i}$ and the sensed signals are fed into the entry point of sensors, i.e., the entry point of the attention selector $T$. In order to recall the effects of the voluntary actions, not only the expected reward value is estimated by the value system, but also the primed context which includes the primed action and the primed sensation.

The architecture illustrated in Fig. 2 is a Type- 4 architecture. Two voluntary internal actions are modeled by $E_{i 1}$ for attention selection, and by $E_{i 2}$ for action release. Both internal actions are sensed by the internal (virtual) sensors $S_{i 1}$ and $S_{i 2}$, respectively. The rehearsed external action (not released) is sensed by the virtual internal sensor $S_{i 3}$. With a SASE agent, both external context (sensed by $S_{e}$ ) and internal context (sensed by $S_{i}$ ) are available in the last context $l \in \mathcal{L}$.

In many engineering models using MDP or POMDP, only the context-relevant actions are evaluated, not the primed (predicted) sensation. The Type- 4 architecture not only enables the primed sensation to be recalled, but also feeds the primed sensation into the perceptual entry port $T$ for further processing. For example, if the robot (1) has experienced or been told that water is cold, wet, and will damage its electronics and (2) sees a lot of water in the river, it can consider the action of running into the river, which primes (predicts) the outcome of feeling cold, wet, and experiencing the absence of sensations from its 
electronics. Psychological models of anticipatory learning processes are not sparse (e.g., [3]), but they typically use a symbolic state representation., which prevents a developmental agent to generate internal representation from new experiences. In the observation driven model of Type-4, adaptive vector (clusters) representation is used, which enables reasoning and prediction, as discussed in the following section.

\section{Reasoning, planning and thinking}

As early as the 1950s, in his article "Computing Machinery and Intelligence" [37], Allen Turing raised a very important question: "Can machines think?". However, Turing avoided a definition of thinking. On the other hand, some established researchers pointed out that neural networks cannot perform reasoning [22]. Any computational reasoning process that is based on a pre-designed set of symbolic states (e.g., POMDP) seems remote to brainlike reasoning and thinking. Modeling autonomous reasoning using neural networks is hard without a mental architecture. The goal of this section is to show that the proposed observation driven mental architectures which used neural networks such as IHDR can perform not only reasoning, but also planning. Autonomous reasoning and planning are gateways toward autonomous thinking.

\subsection{Autonomous reasoning}

Through a consecutive time series $t=1,2, \ldots, k$, the composite function $V \circ R$ performs a series of reasoning, represented by the regression sequence:

$s=\left(\left(l_{1}, p_{1}\right),\left(l_{2}, p_{2}\right), \ldots,\left(l_{k}, p_{k}\right)\right)$,

where each regression pair $\left(l_{i}, p_{i}\right)$ is an input-output pair of the composite function $V \circ R, \quad p_{i}=V \circ R\left(l_{i}\right)$, $i=1,2, \ldots, k$. The link between two consecutive regression pairs can be realized by two paths, the external path and the internal path, denoted by $e$ and $i$, respectively. The external path accounts for external actions and the corresponding environmental changes (e.g., hand touching an apple). The internal path accounts for the signal path from internal action generation (e.g., internal action for attention selection or signals from pre-motor cortex) back to the perceptual input point of $T$ (e.g., internal awareness of attention or pre-motor signals).

Using symbols to denote vectors, the reasoning process can be represented by the following composite reasoning sequence:

$s=\left(\left(l_{1}, p_{1}\right),\left[\begin{array}{c}e_{1} \\ i_{1}\end{array}\right],\left(l_{2}, p_{2}\right), \ldots,\left(l_{k}, p_{k}\right),\left[\begin{array}{c}e_{k} \\ i_{k}\end{array}\right]\right)$,

where

$\left[\begin{array}{c}e_{i} \\ i_{i}\end{array}\right], \quad i=1,2, \ldots, k$

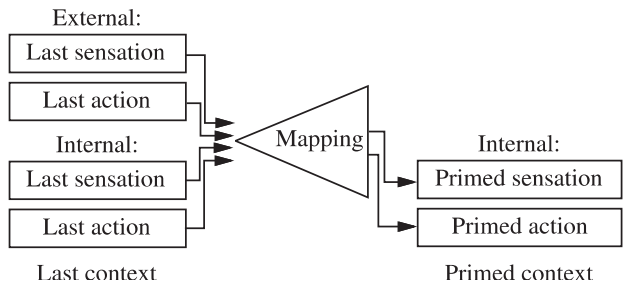

Fig. 5. External and internal reasoning segment. The mapping (left to right) in the figure includes attention selector $T$ and the regressor $R$. The inter-modal attention by $T$ determines whether the corresponding segment in the reasoning process is external, internal, or mixed.

represents the parallel external and internal segments. Whether the result of external and internal segments are taken into account at any time $t$ by the regressor depends on the attention selection in $T$ as illustrated in Fig. 5.

Definition 12 (External and internal reasoning process). There are three types of reasoning processes, external, internal, and mixed, corresponding to the attention in which the attention module $T$ attends to external, internal or both, respectively.

From the above discussion, we have the following summary:

- Type-1-Type-3 architectures allow the agent to perform external reasoning processes, but not internal reasoning as defined above.

- A Type-4 architecture is able to execute external, internal, and mixed reasoning processes.

It is important to note that the internal path is not autonomous thinking if the representation is programmed instead of being autonomously developed from grounded experiences.

Theorem 3. The Type-4 architecture allows internal reasoning to realize the following kinds of learning: (1) nonassociative learning, (2) classical conditioning, and (3) instrumental conditioning.

Proof. First we show for the case of nonassociative learning [32]. The nonassociative learning occurs when the agent is exposed to a series of stimuli because of the history of similar or dissimilar stimuli in the series. Sensitization and habituation are two well-known examples of nonassociative learning. In Eq. (7), the nonassociative learning can be accomplished by the link $\left(l_{i}, p_{i}\right)$ realized by the composition of regression $R$ and the value system: $p_{i}=V \circ R\left(l_{i}\right)$. The value system plays a central role. For example, the action (e.g., looking at another direction) that is predicted to generate more novel stimuli than alternative actions (e.g., continue looking after repeated exposure to the similar stimuli), the former action is selected by the value system $V$ from the alternative actions predicted by $R$.

Next, we prove the case for classical conditioning. In classical conditioning, a conditioned stimulus CS (e.g., tone) is repeatedly paired with unconditioned stimulus US 
(e.g., food) that elicits unconditioned response UR (e.g., salivation). In this case, $l_{i}=\mathrm{CS}, \quad l_{i+1}=\mathrm{US}$, and $p_{i+1}=$ salivation, for all the discrete time instants $i$ where the event occurs. The $Q$-learning used by the value system $V$ back-propagates repeatedly the primed action $p_{i+1}$ through time $i$, so that $l_{i}$ primes $p_{i}=$ salivation even in the absence of $l_{i+1}$.

Finally, we establish the case for instrumental conditioning. When $l_{i}$ stimulus is present, two actions $a_{1}$ and $a_{2}$ are predicted, $\left(a_{1}, a_{2}\right)=R\left(l_{i}\right)$. According to past experience, $a_{1}$ has a low value and $a_{2}$ has a higher value, using, e.g., $Q$-learning by the value system $V$. Thus, $a_{2}$ is selected by $V$.

Fig. 6 illustrates how a signal architecture type- 4 can realize classical conditioning, instrumental conditioning, and other more complex learning modes such as task transfer (where basic skills learned in one context are used in new contexts for more complex perceptual behaviors). From the figure, we can see that the basic mechanism that enables these various types of learning by a single generalpurpose architecture is the priming or prediction: from the last context (including sensation and action) to predict the primed context (including sensation and action).

Instrumental conditioning has been known as reinforcement learning in the machine learning community and has been very widely studied using the traditional MDP architecture [14,34]. A major contribution here is that a single architecture realizes all three types of learning.
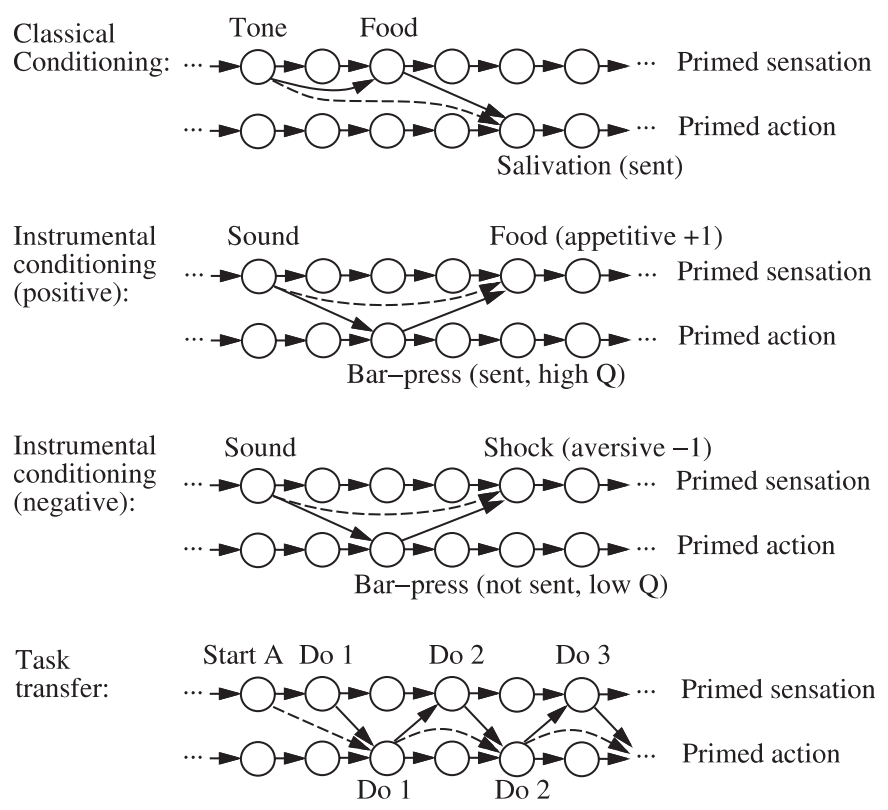

Fig. 6. The single Type- 4 architecture enables classical conditioning, instrumental conditioning, and task transfer. Each circle in the figure indicates a mental state, represented by the best matched ("resonate") context in the long term memory $L$. From left to right is the passing time. Solid arrows denote short-time-separation associations. Dashed arrows denote autonomously learned association through primed contexts (including sensations and actions).

\subsection{Autonomous planning}

There are many more complex internal mental activities enabled by the Type- 4 architecture. As an example, we address a complex activity known as autonomous planning. Planning has been conducted extensively using the traditional MDP architecture, based on the $Q$-learning mechanism (i.e., time discounted value propagation) [41]. However, $Q$-learning based planning has a major drawback: It prefers immediate small rewards to future large rewards. One can program the planner in such a way so that only the final goal produces a reward and intermediate goals do not. However, such a task-specific setting is too inflexible for the general setting of mental development, where various rewards are generated from the real world at different stages and it is impossible for the programmer to write a different program for a different planning task (due to the task non-specific nature of AMD discussed in Weng et al. [47]).

Theorem 4. The Type-4 architecture allows internal reasoning to realize autonomous planning.

Proof. Autonomous planning requires first an accumulation of experiences so that alternative condition-action pairs are learned. Suppose that there are two plans according to the experiences: the execution path of the plan (a) is recalled as

$\left.\left(l_{1}, p_{a, 1}\right),\left(l_{a, 2}, p_{a, 2}\right), \ldots,\left(l_{a, i}, p_{a, i}\right)\right)$

and that of the plan (b) is recalled as

$\left.\left(l_{1}, p_{b, 1}\right),\left(l_{b, 2}, p_{b, 2}\right), \ldots,\left(l_{b, j}, p_{b, j}\right)\right)$.

Both lead to a completion of the task. Both plans are recalled sequentially using only the internal path $i$, instead of the external path $e$ in Eq. (7). Finally the value of $p_{a, i}$ is compared with that of $p_{b, j}$. The value system decides which value is better and so chooses the corresponding plan (a) or (b). The association of $a$ to the primed action in $p_{a, 1}$ and $b$ with that in $p_{b, 1}$ is represented by "talking to itself": for example, the selected plan in $p_{a, i}$, as part of the last context in $l$, primes the first action in $p_{a, 1}$. The similar process takes place for plan (b).

I expect that early demonstration of autonomous planning is possible in a restricted (simplified) natural setting. Anywhere any-time planning in uncontrolled natural settings is possible after a significant amount of "living experience." It is important to note that attention (intra-model and intermodal) at each sensorimotor path plays a powerful role of generalization for new and unexpected settings (see the task-transfer and action chaining in [54]).

One might think at this point that the internal process looks like "thinking." Numerous publications have loosely discussed human thinking (e.g., [33]) and machine thinking (e.g., $[37,26,13])$. The proposed architecture Type-4 allows autonomous thinking. However, the architecture Type-4 
alone is not sufficient for autonomous thinking. A necessary condition for autonomous thinking is AMD, as explained below. In other words, human-like thinking skills are autonomously developed through experience, instead of being totally innate or being programmed-in taskspecific rules.

\section{Type-5: Developmental observation-driven SASE MDP}

Definition 13 (DOSASE MDP). The developmental observation-driven SASE MDP (DOSASE MDP) has an architecture Type-4 or higher, that satisfies the following requirements:

1. During the programming time, the tasks that the agent will learn are unknown to the programmer.

2. The agent $A(t)$ starts to run at the birth time $t=0$, under the guidance of its developmental program $P_{d}$. After the "birth," the brain of the agent is closed: neither readable nor writable to human teachers.

3. Human teachers can only affect the agent $A(t)$ as a part of its environment through its sensors and effectors recursively: at any time $t=0, t=1, \ldots$, its observation vector at time $t$ is the last context $l(t)$. The output from $A(t)$ at time $t$ is its selected primed context $p(t) \in \mathcal{P}$. $A(t-1)$ is updated to $A(t)$, including $T, R$ (and $L$ ), $M$, and $V$.

Because of the task nonspecificity, the DOSASE MDP $\left(A(t), P_{d}\right)$ does not require a given set of states that have task-specific meanings. In contrast with the traditional MDP, consequently, $P_{d}$ does not require a given estimate for the prior distribution probabilities $P\left(l_{0}\right)$, state transition probabilities $P\left(l_{t} \mid l_{t-1}\right)$, and the state observation probabilities $P\left(x_{t} \mid l_{t}\right)$, since such probabilities are meaningful only after their task-specific meanings are given.

When the number of states is very large, it is practically sufficient to only keep track of the states that have a high probability, instead of estimating the probability of all the states, which is too computationally expensive to reach the real-time speed.

\section{Type-6: Multi-level DOSASE MDP}

The Type-5 architecture has only one sensory level, although each mapping inside the level (i.e., the attention selector $T$, the regressor $R$ ) has multiple levels in its own internal structure. We call it a sensorimotor level because the pathway from $T$ through $R$ up to $M$ corresponds to a pathway from sensory input to motor output.

Such a single sensorimotor level corresponds to a certain degree of sensory integration, integrating different receptors of a single modality (e.g., vision) shown as the lowest modules on the left side of Fig. 3.

\subsection{Why multi-level}

However, a single modality is not sufficient to describe the architecture of the brain, because it is able to jointly process multiple sensory modalities (e.g., audition and lipreading). Fig. 7 illustrates how multiple sensory modalities are integrated (e.g., vision and audition). Considering three sensory modalities, vision, audition and touch, the brain devotes certain cortical area for every two-modality (bimodal) combination, visual-auditory, visual-touch, and auditory-touch. The cortical size devoted to every particular bimodal combination is adaptive in competition through experience. Further, bimodal sensory processing enables three-modality (trimodal) sensory integration, as illustrated in Fig. 7 (e.g., visual-auditory-touch integration).

Likewise, separate control of individual every single motor (e.g., muscle) is not sufficient to describe the architecture of the brain. Different muscles often work together (e.g., walking). Therefore, a motor hierarchy is necessary, shown as the motor hierarchy on the right side of Fig. 3. Like the sensory hierarchy, the motor hierarchy integrates motors according to innate predisposition and their postnatal experience of coherent movements. The motor hierarchy, the control signals from motors are used as input signals for the development of motor cortex.

\subsection{Sensory and motor hierarchies}

For effective use of limited cortical source, the brain dynamically assigns cortical areas to the sensory hierarchy and the motor hierarchy. The complete mechanisms that determine the resource relocation are not clear at this point. It is expected that the frequency of occurrence of

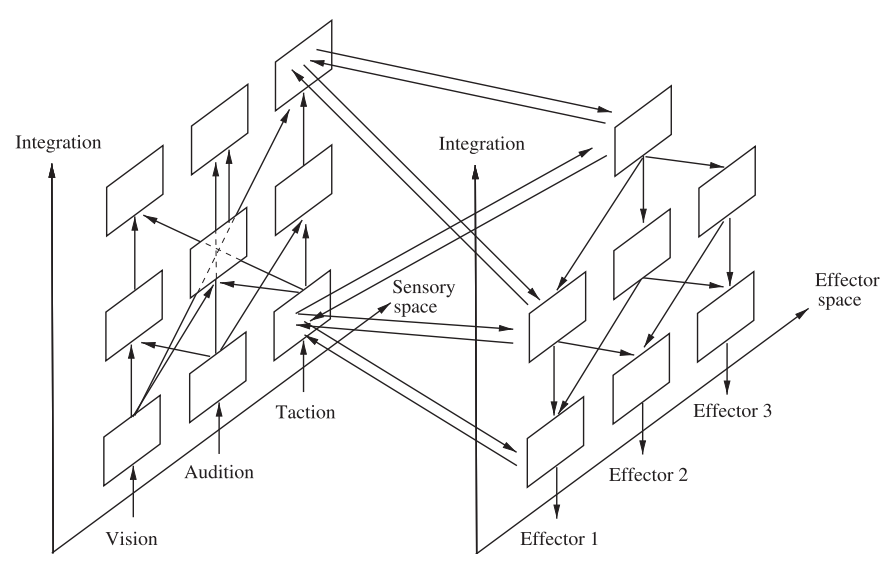

Fig. 7. The two-hierarchy mental architecture: the sensory hierarchy (on the left side) and the motor hierarchy (on the right side). A left-to-right arrow from the sensory hierarchy to the motor hierarchy indicates association from the integrated sensory group to the integrated motor group (e.g., walking). A right-to-left arrow from the motor hierarchy to the sensory hierarchy indicates a feedback flow of internal action per SASE model, such as attention selection executed by the sensory mapping or the feedback of action as a part of internal sensory input. 
a particular combination and the associated attention to the current particular combination may play a role in the competition for cortical resources.

The above discussion amounts to the two-hierarchy architecture illustrated in Fig. 3, which includes a sensory hierarchy and a motor hierarchy. Through experience, higher modules are developed gradually in both the sensory hierarchy and the motor hierarchy. During early learning, the links between the sensory hierarchy and the motor hierarchy tend to be from lower sensory modules to lower motor modules. That is why it is difficult to generate skilled actions during early learning (e.g., walking).

With the development of higher sensory modules and higher motor modules, as illustrated in Fig. 7, projections from higher sensory modules to higher motor modules are also gradually generated, representing skilled stereotypical sensorimotor behaviors that integrate more sensors and effectors (e.g., arm-leg coordination during walking). With the process of such development, complex sensorimotor behaviors that originally require intensive attention on the part of the learner gradually become automatic, largely attention-free sensorimotor behaviors. From infancy to adulthood, this developmental process takes place for all the sensorimotor behaviors, including walking, visual and speech recognition, language understanding, speech production, situation awareness and common sense.

It is important to note that the association between the sensory hierarchy and the motor hierarchy is not any taskbased hierarchy (e.g., a higher level solves a multi-city trip task and a lower level solves a single-city trip task). The developmental brain is not able to divide its cortical regions strictly according to tasks.

It is also important to note that the two-hierarchy architecture in Fig. 7 is not exactly the same as the behavior-based vertical robot architecture of Rodney Brooks [6] either, e.g., path planning at a higher level and collision avoidance at a low level. Brooks did an excellent job in adapting neural science motivated ideas into intuitive behavior-based concepts in robotics. However, the developmental brain is not able to divide its cortical regions strictly according to human intuitively understandable behaviors. The scale of sensory and effector integration is indirectly related to behavior sophistication, but not directly. Further, it requires not only motor integration but also sensory integration.

On the basis of cytoarchitectonic and cortical-connection data, Pandya and Seltzer [25] proposed a refined division of cortex: primary, association, multimodal, and paralimbic cortices. Such a division cuts across both the sensory hierarchy and the motor hierarchy outlined here. The sensory mapping $T$ roughly corresponds to what Pandya and Seltzer [25] called primary cortex; the cognitive mapping $R(L)$ with the distributed value system $V$ are closely related to what they called association cortex. The paralimbic cortex belongs to the motor mapping $M$. The multimodal cortex is explained in the second level (and above) on the sensory hierarchy shown in Fig. 7.
A key question for building a sensory hierarchy is what information is fed from a low-level sensorimotor system to a higher level sensorimotor system (e.g., what is fed from the vision module to the visuoauditory module). As we discussed, the level indicates the scale of receptors being integrated. Of course, it will be wasteful for the brain to simply devote a new cortical area to directly process the larger scale of receptors to be integrated (e.g., all the visual receptors and all the auditory receptors cells). Instead, the response (i.e., feature values or associated context) from the corresponding lower-level (smaller scale) modules should be used as input to the higher level. As indicated in Fig. 7, two types of information are needed as sensory input to a higher level:

1. Primed context (or response) from the low level modules (cognitive mappings) as in Fig. 7 as a flow from low to high in the sensory sub-hierarchy.

2. The projections from other areas, such as the internal actions for attention selection, shown as right-to-left arrows in Fig. 7.

These types of information are needed for the following major reasons:

1. Enabling a higher degree of sensory integration. When vision and audition are combined at the higher level, lip reading can be performed to disambiguate auditory input in a noisy environment [19]. The primary cortex for such joint visual and auditory information is called a bimodal cortex. A bimodal cortex is typically significantly smaller than the corresponding unimodal cortices.

2. Cross-modal and intra-modal attention. Cross-modal attention and intra-modal attention deal with attention choices between different modalities (e.g., vision and audition) and different receptive fields in the same sensing modality, respectively. Modularity can reduce the task of attention selection.

3. Self-generated internal context: allow voluntarily generated motor actions to serve as context input to other modules. Thus, the agent is able to "talk to itself".

Definition 14 (Type-6). The Type-6 mental architecture is composed of several levels of Type- 5 architecture. The primed contexts (or response) from a lower-level system are fed into the sensory input of the higher-level system.

Fig. 8 illustrates the Type- 6 architecture. The input to the attention selector $T$ at level 2 includes the primed context $p(t)=\left(x_{p}(t), a_{p}(t)\right)$ from level 1 , where $x_{p}(t)$ and $a_{p}(t)$ are primed sensation and primed action, respectively. One or multiple levels can feed their primed contexts into the next higher level for sensory integration.

\subsection{Discussion}

We have systematically introduced six types of architectures. Although the order at which new capabilities are 


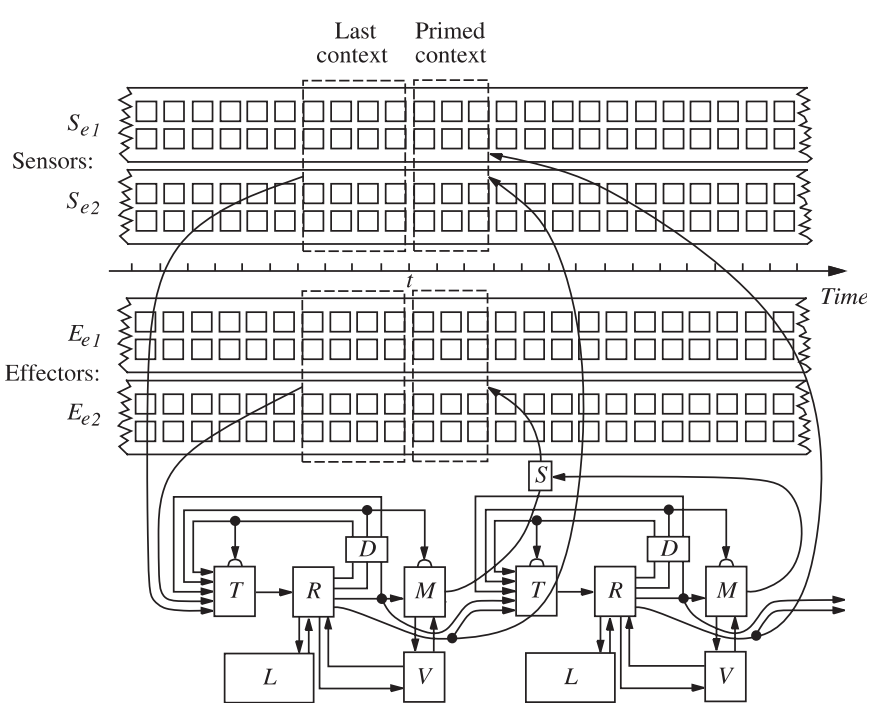

Fig. 8. The Type-6 architecture: multi-level DOSASE MDP.

added to the previous type is primarily a design choice, the order used here is motivated by a relatively large payoff in capability with a minimal addition to the architecture complexity.

At the conclusion of the above exposition of a series of architectures, let us back off to examine the major goal of a theory of mental architectures. Although many factors are involved in considering mental architectures, a major goal is to enable superior performance with a limited amount of training or exploration, namely, generalization. Three types of generalizations are involved: architecture design driven, innate value driven, and learned value driven.

The architecture design driven factors include: (1) The mechanism of the bottom-up feature extraction (sensory mapping) and the top-down attention selection. (2) The mechanisms of discriminant analysis and quasi-optimal statistics based representation generation in early processing (e.g., in attention selector $T$ ) and later processing (e.g., in regressor $R$ ). (3) The mechanism of abstraction from lower level (more space and time related) to higher level (coarser and more flexible in space and time).

The innate value driven factors include: (1) Pleasure seeking and pain avoidance: the system will repeat contextsensitive actions that lead to rewards and avoid actions that lead to punishments. (2) Novelty seeking: with similar expectation of pleasure and pain, the system tends to adopt context-sensitive actions that lead to higher novelty (e.g., the high difference between the actual sensation and the predicted sensation).

The learned value driven factors include: (1) Attention selection: the learned attention behaviors will enable the system to pay attention only to the parts that are related to the task at hand, which disregard unrelated inputs and facilitate generalization in different but partially similar settings. (2) The longer-term prediction enabled by multilevel architecture, which will allow the system to learn what is the right thing to do and what is not, and further, the criteria of success (e.g., behavioral or moral criteria). Of course, many of these mechanisms have yet to be further studied and demonstrated.

\section{Refined learning types}

Although a model of architecture can be valid in its own right without necessarily being accompanied by ways in which the architecture enables its autonomous learning (development), the associated theory of learning is important to better understanding the architecture and to guide its applications.

In the machine learning literature, there have been widely accepted definition of learning types, such as supervised, unsupervised, and reinforcement learning. However, these conventional definitions are too coarse to describe computational learning through autonomous development. For example, it is difficult to identify any type of learning that is completely unsupervised. Traditional reinforcement learning in machine learning is too restrictive - it does not allow supervised learning of any sort and does not distinguish sensors that have congenital preference patterns and those that do not. Further, the traditional classification of animal learning models, such as classical conditioning and instrumental conditioning, is not sufficient to address computational considerations of every time instant of learning (e.g., the fine time scales of all combination of related factors). A definition of a refined classification of learning types is necessary.

\subsection{Biased and unbiased sensors}

To distinguish the channels of reward (e.g., sweet and pain sensors) that are available at the birth time, and other channels of reward that are not ready to be used as reward at the birth time (e.g., auditory input "good" or "bad"), we define congenitally biased sensors:

Definition 15 (Congenitally biased sensors). If the machine has a congenital preference for some sensory patterns from a sensor, this sensor is an congenitally biased sensor. Otherwise, it is an congenitally unbiased sensor.

We may drop the term "congenitally" when the context is clear. Due to the sophistication of the processes of geneexperience interplay throughout prenatal and postnatal development, currently it is very difficult to identify biological sensors (receptors) that are truly unbiased at the birth time. The situation is different with machines, since a programmed direct mapping between the signals of a sensor to the computed values can identify such biased sensors. For example, a robot can have a set of "good" buttons and a set of "bad" buttons. Each "good" or "bad" button corresponds to the value of a particular effector. The forward bumper sensor is a "bad" button that is associated with forward move effector.

It is important to note that all the sensors become biased gradually through postnatal experience- the development 
of the value system. For example, the image of a flower does not give a newborn baby much reward, but the same image becomes pleasant to look at (high value) after the baby has grown up.

\subsection{Eight learning types}

To more clearly define various learning types and to eliminate the implicit limitation imposed by traditional machine learning types, I introduce a refined definition of learning types, using three factors.

The first factor denoted by a variable $i$, indicating internal task-specific representation, distinguishes the difference between hand-crafted task-specific internal representation and biologically inspired AMD which is free of gene-coded (hand-crafted) task-specific internal representation. $i=1$ indicates that at least some internal task-specific representation is imposed by a human programmer (called internal-state imposed $i=1$ ). Otherwise, $i=0$ (called internal-state autonomous $i=0$ ).

The second factor that is essential for the classification of learning types is the autonomy of effector, denoted by a variable $e$. If the concerned effector is directly guided by the human teacher or other teaching mechanisms for the desired action, we call the situation action imposed $(e=1)$. Otherwise, the learning is effector autonomous $(e=0)$.

The third factor for the classification of learning is whether or not a biased sensor is used. A variable $b$ is assigned for denoting biased sensors. If any biased sensor is activated (changed its value, increasing or decreasing) during the learning, we called the situation reinforcement $(b=1)$. Otherwise, the learning is call communicative $(b=0)$.

Using these three key factors, any type of learning can be represented by a 3 -tuple $(i, e, b)$, which contains three components $i, e$, and $b$, each of which can be either represented by 0 or 1 . Thus, there are a total of eight different 3-tuples, representing a total of eight different learning types. If we consider ieb as three binary bits of the type index number of learning type, we have eight types of learning defined in Table 1 . We can also name each type verbally. For example, Type 0 is state-autonomous, effector-autonomous, communicative learning. Likewise, Type 7 is state-imposable, effector-imposed, reinforcement learning.

In using the above definition, the factors should include all entities that are related to the task being learned. For example, if a sweet food is introduced to the mouth of an autonomous animal while its trainer says "good job!", the learning type is Type-1, although biased sensors and unbiased sensors are both used. This is because it is almost impossible to identify a learning case where none of the unbiased sensors is used. Of course, detailed sets of sensors and effectors may be necessary to fully specify a learning process. For example, some effectors are imposed while other effectors are autonomous.
Table 1

Eight types of learning

\begin{tabular}{llll}
\hline Type (binary) & Internal state & Effector & Biased sensor \\
\hline $0\left(\begin{array}{llll}0 & 0 & 0\end{array}\right)$ & Autonomous & Autonomous & Communicative \\
$1\left(\begin{array}{lll}0 & 0 & 1\end{array}\right)$ & Autonomous & Autonomous & Reinforcement \\
$2\left(\begin{array}{lll}0 & 1 & 0\end{array}\right)$ & Autonomous & Imposed & Communicative \\
$3\left(\begin{array}{lll}0 & 1 & 1\end{array}\right)$ & Autonomous & Imposed & Reinforcement \\
$4\left(\begin{array}{lll}1 & 0 & 0\end{array}\right)$ & Imposable & Autonomous & Communicative \\
$5\left(\begin{array}{llll}1 & 0 & 1\end{array}\right)$ & Imposable & Autonomous & Reinforcement \\
$6\left(\begin{array}{lll}1 & 1 & 0\end{array}\right)$ & Imposable & Imposed & Communicative \\
$7\left(\begin{array}{lll}1 & 1 & 1\end{array}\right)$ & Imposable & Imposed & Reinforcement \\
\hline
\end{tabular}

\subsection{Discussion}

According to this new kind of classification, all learning types using a non-developmental learning method correspond to Types 4-7, this is because the task-specific representation is handcrafted after the task is given. AMD uses Types $0-3$.

We first consider traditional machine learning Types 4-7, where the internal states are hand-crafted based on a given task, such as the non-ODMDP. The traditional supervised learning corresponds to Type 6 and the traditional reinforcement learning corresponds to Type 5 . The operational mode of type 4 is typically called test, not learning. Type 7 has been rare in machine learning.

Next, we consider developmental learning Types 0-3, where the internal states are not hand-crafted based on any tasks, since tasks are not given during the time of programming (or before the birth). Types 3 and 2 enable the teacher to manually guide the effector of the learner, e.g., the teacher holds the hand of a child and guides it to teach the child how to draw. With Type 3, the teacher also gives physical rewards to the learner, e.g., giving him candy. However, types 3 and 2 are too tedious for autonomous learning, since it requires a human teacher to specify exact output in real time. Therefore, it is possible only for a limited time for some small number of effectors. Furthermore, internal actions cannot be taught using type 3 or 2 , since there is no way for a teacher to directly impose an internal action.

Type 1, state-autonomous, effector-autonomous reinforcement learning, enables the teacher to teach internal behaviors. For example, when the robot looks at the teacher, the teacher presses its "good" button, which reinforces the attention selection behavior. However, Type 1 learning is not very effective for more general settings. First, the number of all possible actions to explore is simply too large to try them all. Second, there is the credit assignment problem. For example, when the teacher gives the baby a candy, is the reward due to the fact that the baby looks at the teacher or that she is simply being quiet? The following new mode of learning can drastically reduce the complexity of autonomous learning, for both internal and external actions. 


\subsection{Communicative learning}

The most powerful learning mode, and it is the major mode of learning for human adults, is Type 0, stateautonomous, effector-autonomous communicative learning. On the surface, it is not very obvious to see how learning takes place in the Type 0 mode. This requires a new type of learning called communicative learning introduced in Weng 2004 [43]:

Definition 16 (Communicative learning). Communicative learning is achieved through the Type-0 learning mode. However, Type-0 learning mode requires two processes, which can be interwoven through development: (1) Grounded language acquisition (using any mode of learning). (2) Teaching using an acquired language (verbal, written, sign, etc.).

In the definition, grounded language acquisition is important because the meaning of the language is directly associated with physical senses and actual actions. Depending on the sophistication of the meaning of the language used (words, phrases, or a full natural language), teaching using language is often significantly more effective than other learning types. For example, we will see in next Section 10.4 how the self-organizing autonomous incremental learner (SAIL) robot acquired grounded meaning of each verbal command first and then used acquired language (verbal commands) to learn how to draw a flower in a new task setting.

\section{Experimental studies}

All the learning experiments presented here were conducted incrementally (about $30-100 \mathrm{~ms}$ per mental cycle) online in real-time, except those stated otherwise. As stated, the representation of the architecture must be task-nonspecific. Raw high dimensional video frames were used as input vectors. An image of $m \times n$-pixels is converted into a vector of dimension $d=m n$, where each pixel corresponds to a component (receptor). The mean and variance of the entire image frame are normalized to be zero and unit, respectively, so that the image is insensitive to the illumination level and contrast. The biologically inspired Mel Frequency Cepstral Coefficients (MFCC) were used as auditory input, simulating to some degree the hair cells in the (auditory) cochlea. All the touch sensors are represented by their range-normalized values. Every effector is represented by its per-effector normalized speed or position values. There is no task-specific feature extraction in the design of the developmental program. Therefore, all the signals can be used in other tasks.

The "brain" of the SAIL robot is totally signal driven (from sensors and effectors), generated by the SAIL developmental program based on the type- 6 architecture. There is no need for initial guess (e.g., weights of connections), since early data are used to initialize the modules. In the experiments described here, supervised learning (like pushing a wheelchair during learning visionguided navigation) was used to generate some desired behaviors. Boltzmann exploration [34] was used to generate early value-guided pseudo-random exploration.

SAIL's value system was developed using the method described in [10]. Each primed context on the left side of Eq. (1) is assigned a $Q$ value, computed by the $Q$-learning algorithm [41]. The reward at any time is a weighted sum of three types, aversive stimulus, appetitive stimulus, and novelty, with the highest weight for the aversive stimulus and the lowest for novelty. The novelty is the normalized Euclidean distance between the primed context vector and the actually sensed context vector. The normalization is to divide each component in the vector by the temporally incrementally computed averaged standard deviation, so that the degree of expected disparity between the expected and actual value is taken into account. Movies are available at http://www.egr.msu.edu/ $\sim$ weng/research/ LM.html.

\subsection{Attention selector $T$}

The purpose of this experiment, illustrated in Fig. 9, is to show the need for attention selector $T$. Without it, the regressor $R$ is forced to do global template matching, which is fine in theory as long as enough samples are available to densely fill the space of global patterns. However, when related patterns are all occluded in learning, the space of global patterns is not filled densely. Active attention executed by attention selector $T$ enables local analysis which concentrates on a subspace of the global sensory space. This subspace, represented by partial views, has a lower dimension and is filled densely. The learning for attention control and label output used Type-2 learning mode.

We have designed and implemented an attention selector $T$ in Fig. 2 as a part of what is more general called sensory

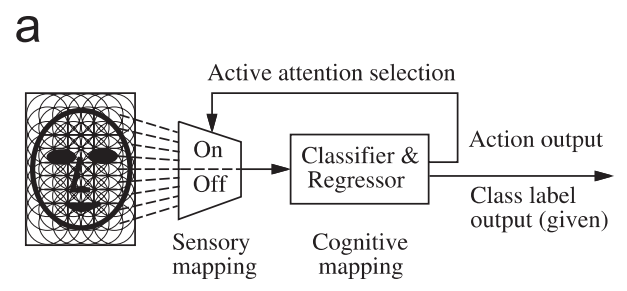

b

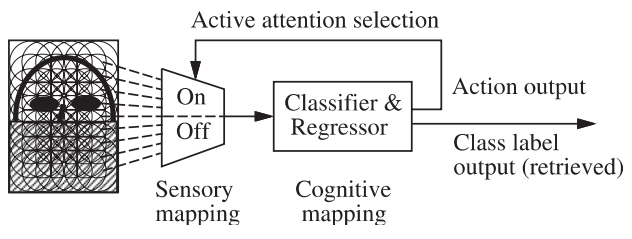

Fig. 9. Active attention during learning and performance sessions enables recognition of occluded faces. (a) Learning session. (b) Performance session. 
mapping. A sensory mapping simulates the role of early sensory processing (such as the retina, LGN, V1, and V2). A model of sensory mapping, called "Staggered Hierarchical Mapping" (SHM) [51] consists of several layers of inter-connected neurons, providing representation for receptive fields at different retinal locations and of different sizes. It was automatically developed from the agent's visual experiences. In addition to the role of attention selector $T$, the sensory mapping also accomplishes the role of feature detection, which in turn serves the role of dimension reduction from input while retaining necessary information in the input.

The regressor $R$ was implemented by the hierarchical discriminant regression (HDR) [12,44], as shown in Fig. 9. The experiment was organized as follows: in the training session, a series of nonoccluded face images were presented to the system with class labels (name of the person). The system takes upper (U) and lower (L) (roughly half) views, controlled by the supervised attention control. In other words, to concentrate on the evaluation of SHM model for $T$ in this experiment, we assumed that the desirable attention signal ( $\mathrm{U}$ or $\mathrm{L}$ ) is supplied by the human teacher.

The regressor $R$ (called cognitive mapping) learns two action outputs from the currently sensed image, (1) the required attention selection (upper or lower view) and (2) the class label of the face image. In the performance session, the learned attention control behavior controls the attention selection via SHM sensory mapping, which feeds the response to the following IHDR cognitive mapping.

If only the upper view is available (not occluded), the result is called U. Similarly, if only the lower view is available (not occluded), the result is called L. If the system feeds the upper view and the lower view as an integrated long response vector into the IHDR classifier (upper view and lower view is occluded individually at two consecutive views), the result is called $\mathrm{U}+\mathrm{L}$.

The role of $T$ is clear: if a system is passive (without active attention selection), it learns the global view (not occluded) but in the performance session it tries to match the input (occluded) $\mathrm{U}$ or $\mathrm{L}$ view with the learned globalview (not occluded) prototype. This is called monolithic vision (i.e., global template matching).

In this experiment, the sensory mapping SHM was developed from viewing over 5000 natural images [51]. Attention control is an internal action (acting on the "brain"), which normally does not allow supervised learning (the internal effector is not accessible from the environment). Since reinforcement learning takes a significant amount of training time, we used supervised learning to speed up the learning in this study.

To develop the regressor $R$ for the face recognition task, we used a face data set from the Weizmann Institute in Israel. The data set was taken from 28 human subjects, each having 30 images with all possible combinations of two different expressions, three lighting conditions and five different facial orientations. The result is summarized in Table 2, where if we use the nearest neighbor method (NN)
Table 2

Summary of recognition under occlusion

\begin{tabular}{llll}
\hline Method & \multicolumn{3}{l}{ Recognition rate } \\
\cline { 2 - 4 } & $\mathrm{U}$ & $\mathrm{L}$ & $\mathrm{U}+\mathrm{L}$ \\
\hline Monolithic + NN & $51.43 \%$ & $75.83 \%$ & $82.38 \%$ \\
SHM + HDR & $92.86 \%$ & $95.95 \%$ & $98.57 \%$ \\
\hline
\end{tabular}

for the monolithic vision case to find the prototype, the result is called monolithic $+\mathrm{NN}$. The result clearly demonstrated the necessity of active attention using the sensory mapping, whose recognition rate (SHM + HDR for $\mathrm{U}$ and $\mathrm{L}$ cases) is significantly higher than the case without sensory mapping (Monolithic $+\mathrm{NN}$ for $\mathrm{U}$ and $\mathrm{L}$ cases) in the presence of occlusion.

In this study, the internal attention selection actions during training were programmed using a predetermined rule. In another study, Huang and Weng [11] provided a model of a developmental value system and showed how the attention selection signals were generated autonomously.

\subsection{Learning regressor for vision-guided navigation}

In this study, we concentrated on regressor $R$ developed by the IHDR method [44], using Type-2 learning mode. The attention selector $T$ was not included due to its demand of high computation power. Although attention selection could be useful for vision-guided navigation, we used a monolithic global view in this study of a learning regressor. In the experiment of vision-guided navigation [46], a human teacher taught the SAIL robot by taking it for a walk along the corridors of MSU's Engineering Building. Force sensors on the robot body sense the push action of the teacher and its two drive wheels comply by moving at a speed that is proportional to the force that is sensed on each side. In other words, the robot performed supervised learning in real-time.

In the experiment, after four trips along slightly different trajectories along the corridors, the human teacher started to let the robot "go free." He needed to "hand push" the robot at certain places, when necessary, until the robot could reliably navigate along the corridor, without a need for "hand-leading." We found that about 10 trips were sufficient for the SAIL robot to navigate along the corridors, using only vision, without using any range sensors. Fig. 10 shows some images that the robot saw during the navigation.

Since the processor can be generated automatically "on the fly," the SAIL robot was moved outdoors (around the Engineering Building) for autonomous navigation, without the need for reprogramming, and has made a limited success [52]. 

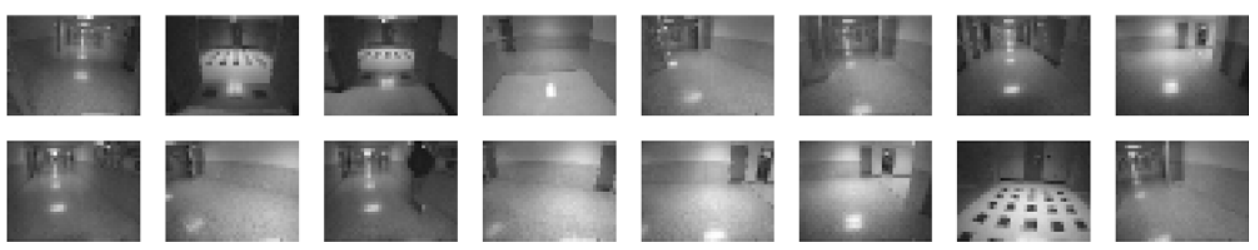

Fig. 10. A subset of images sensed by the SAIL robot in the autonomous navigation, showing the wide variation of the scenes that the robot needs to learn.

\subsection{Developmental audition}

To show the generality of the architecture, we also conducted experiments for speech recognition. Our developmental speech learning is very different from the traditional training of speech recognition systems [40,31] (e.g., IBM's ViaVoice or Dragon's naturally speaking products) in the following sense: (1) The continuous auditory streams have not been segmented and labeled (thus, autonomous learning is possible). (2) During learning, the entire auditory system must listen to everything (for autonomous learning), in contrast to traditional supervised learning where each single designed model (e.g., a single HMM word model) listens to only segmented speech corpora of the single class that it is designed to recognize (e.g., various utterances of the word "forward"). That is, developmental auditory learning is a much harder problem. (3) No syntax is involved during programming (e.g., the system can learn words and phrases from multiple languages concurrently). The above points (1) and (2) are necessary for autonomous speech learning. No traditional speech recognition methods can deal with them. Point (3) is necessary for the task-nonspecificity nature of development.

The SAIL robot learned to follow voice commands through physical interaction with a human trainer [53]. In the early supervised learning stage (Type-2 learning mode), a trainer spoke a command (a word or a continuous phrase) to the robot and then executed a desired action by pressing a pressure sensor or a touch sensor that was linked to the corresponding effector. In later stages, when the robot could explore more or less on its own, the human teacher used reinforcement learning by pressing its "good" or "bad" button to encourage and discourage certain actions (Type-1 learning mode). Typically, after about 15-30 min of interactions with a particular human trainer, the SAIL robot could follow commands with a correct rate of about $90 \%$. Table 3 shows the voice commands learned by the SAIL robot and its online test performance. Later, when the teacher teaches the robot to navigate by using the learned verbal commands (e.g., left and right), the learning mode is Type- 0 .

In other words, the robot performed grounded, autonomous early language acquisition (words and phrases only so far without complex syntax) and later used the simple language for learning navigation (i.e., communicative learning). This open-ended learning was impossible before
Table 3

SAIL robot's performance in developmental audition

\begin{tabular}{llllll}
\hline Commands & Go left & Go right & Forward Backward & Freeze \\
\hline Correct rate (\%) & 97.1 & 91.3 & 93.8 & 100.0 & 80.0 \\
No. of tests & 35 & 23 & 65 & 7 & 5 \\
Commands & Arm left & Arm right & Arm up & Arm down Hand open \\
Correct rate (\%) & 100.0 & 90.0 & 100.0 & 100.0 & 90.0 \\
No. of tests & 10 & 10 & 10 & 10 & 10 \\
Commands & Hand close See left & See right See up & See down \\
Correct rate (\%) & 90.0 & 100.0 & 100.0 & 100.0 & 100.0 \\
No. of tests & 10 & 10 & 10 & 10 & 10 \\
\hline
\end{tabular}

with traditional approaches. It is not simply to link a symbolic command to a symbolic action, but real-time incremental associations from very complex feature regions to desired actions. The feature regions are complex because phrases do not have a fixed or marked temporal boundary, spoken temporal waves are time-warped differently in each utterance (different temporal parts are slowed differently when you speak at different times) and auditory features are very different between speakers.

\subsection{Transfer and chaining}

A more complete architecture in the experiment is illustrated in Fig. 11, which enables a robot to develop complex skills after acquisition of simple ones [54].

Suppose that the SAIL robot was taught to do subtask $T_{i}$ after hearing command $C_{i}, i=1,2,3,4$ (e.g., using Type-2 learning mode). How does the teacher teach it to conduct a more complex task which requires subtasks $T_{1}$, $T_{2}$ and $T_{3}$ consecutively after hearing a new command $C$ ?

As explained above, the major architecture mechanism that makes this possible includes priming and attention, one that realizes chained secondary conditioning. The last case in Fig. 6 explained how the architecture enables task transfer: the sensorimotor skills learned in previous experience of performing simpler tasks are used to learn to accomplish more complex tasks in later experience.

The learning type used here is communicative learning (Type-0). In the language-acquisition phase, the SAIL robot learned to associate context $C_{i}$ with action output $A_{i}$ :

$C_{i} \rightarrow A_{i}$, 


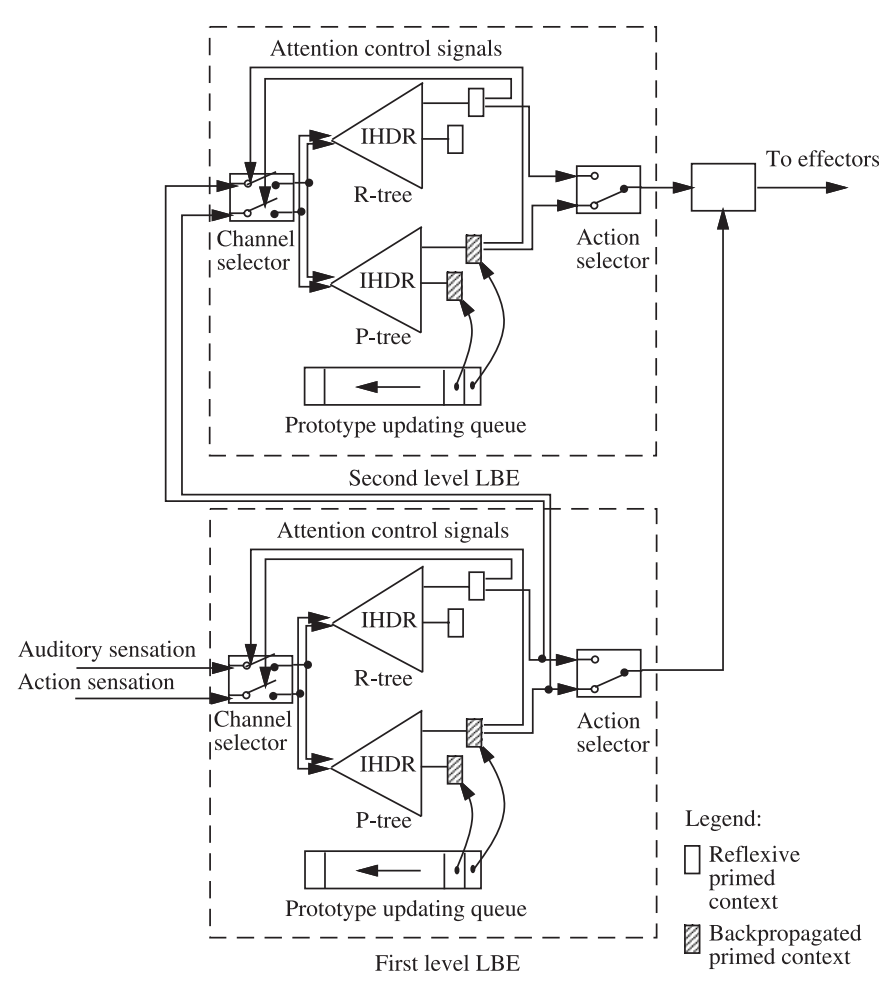

Fig. 11. An integrated type-6 architecture with two sensorimotor systems, one lower (less abstract) and the other higher (more abstract) to accomplish developmental learning for more complex skills such as transfer and chaining.

where $\rightarrow$ means "followed by," $C_{i}$ is a (verbal) command spoken in a natural setting, and $A_{i}$ is a sequence of desired sensorimotor sequences, $i=1,2,3,4$. In this language acquisition phase, any type of learning can be used. In the experiment, we used Type-2 learning: state-autonomous, effector-imposed, and communicative. This is done by stating a verbal command and then guiding the compliant robot arm (through micro-switches) to perform the corresponding action. $C_{1}, C_{2}, C_{3}$, and $C_{4}$ correspond to auditory inputs "upper," "lower," "left," and "right," respectively. $A_{1}, A_{2}, A_{3}$, and $A_{4}$ correspond to the desired motor sequences to draw the upper, lower, left, and right petals, respectively. This is a process of acquisition of a very simple language in an embodied, incremental, and real-time fashion.

With the same developmental program continuously learning, the next session in the mind of the human teacher is to use the acquired language skills to learn more complex sensorimotor behaviors. For example, the teacher wants to give a new command, e.g., $C$. And, he desires that the robot obeys by executing actions $A_{1}, A_{2}, A_{3}, A_{4}$, consecutively, and then stops. However, the robot has only learned to respond to command $C_{i}$ with action $A_{i}$ and at the end of action $A_{i}$, it stops.

The key in making the transfer possible is to bring the desired contexts into close temporal neighborhood. This is a typical teaching method by providing a hint at the right time. For example, when the teacher states $C$, which the machine does not understand and invokes no action, the teacher next states the verbal command $C_{1}$ immediately so that the verbal command brings the associated and executed action $A_{1}$ into close temporal proximity of $C$, as formulated below:

$C \rightarrow C_{1} \rightarrow A_{1}$.

The priming mechanism of $Q$-learning, through the prototype updating queue after a few repeated practices, back-propagates the context $A_{1}$ through state-transition traces so that the prototype of the context $C$ primes not only the sensory context $A_{1}$ but also action $C_{1}$, denoted as:

$C \rightarrow\left\{C_{1}, A_{1}\right\}$

where $\left\{C_{1}, A_{1}\right\}$ indicates that both contexts, one sensory and the other action, are primed as indicated by the architecture in Fig. 2 or 8. After a few practices, the SAIL robot will recall sensation $C_{1}$ and action $A_{1}$ as soon as it receives sensory context $C$. According to the operation of the architecture, the action $A_{1}$ is executed. This developmental learning process is denoted as

$C \rightarrow C_{1} \rightarrow A_{1} \Rightarrow C \rightarrow A_{1}$,

where $\Rightarrow$ means "develops." This is the second phase of communicative learning: learning using acquired language. This type of learning is drastically more efficient than reinforcement learning since the desired action $A_{1}$ is hinted directly by the human teacher, instead of based on trying all possible actions using, e.g., Boltzmann exploration.

Furthermore, this is not the traditional supervised learning in the following sense: the information about desired output is not fed through the effector channel, but instead through the regular sensory channel, which can contain any other environmental information.

The tail context of $A_{1}$ serves a similar role as $C$ if the teacher states verbal command $C_{2}$ as soon as $A_{1}$ ends. Analogously, after a few practices, we have

$A_{1} \rightarrow C_{2} \rightarrow A_{2} \Rightarrow A_{1} \rightarrow A_{2}$.

An important mechanism that is needed for successful learning is the intermodal attention mechanism: attend to $C_{1}$ or $A_{1}$ in Eq. (8), as illustrated in Fig. 5. The value system for intermodal attention behavior was such that more novel context is attended in the absence of aversive and appetitive stimuli [11]. $A_{1}$ is more novel than $C_{1}$ since its sequence ended later than $C_{1}$.

A major technical challenge of this work is that training and testing must be conducted in the same mode through online real-time successive interactions between the robot and the trainer. Another major challenge is the high dimension of the sensory inputs and the wide variation among speakers.

Upon learning the basic gripper tip movements (Fig. 12), the SAIL robot learned to combine individually instructed movements into a composite one invoked by a single verbal command without any reprogramming (Fig. 13). To handle the "abstraction" issue in real sensory inputs, a multi-level 
architecture was used with the higher level emulating the function of higher-order cortex in biology in some sense.

In this experiment, the process of "arranged experience" is in line with the concept of "zone of proximal development" (ZPD) proposed by Lev Vygotsky [39]. ZPD is a latent learning gap between what a child can do on his or her own and what can be done with the help of a teacher. Wood et al. [50] used the term "scaffolding" to describe such an instructional support through which a child can extend or construct current skills to higher levels of competence. When the children become more skillful, the scaffolding (arranged experience) is slowly removed.

As explained above, the SAIL's multi-level architecture enables the association to take place in longer temporal scales and coarser spatial resolution scales, which facilitates abstraction. Sharing of simpler skills (a form of autonomous chunking) takes place automatically, within a task and across tasks, when developing more complex skills. However, when a robot or child develops increasingly more complex cognitive and behavioral skills, the success of teaching (e.g., category learning such as animals and machines) depends on many factors (e.g., attention of certain parts of objects or similarity of subwords). The presented mental architecture, although general, needs the success of all required basic skills at the time of learning. However, mistakes can be corrected in subsequent learning.

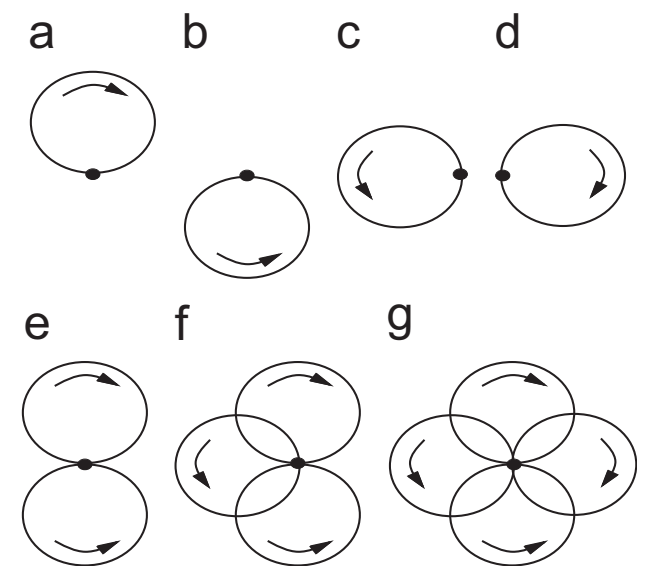

Fig. 12. Autonomously learning how to draw a flower: from simple to complex. (a)-(d) are basic actions, each of which starts from the black dot. (e)-(g) are composite actions achieved by transferring and chaining some or all of the basic ones.
Due to space constraint, some other architectural experiments cannot be described here, e.g., SAIL's object permanence experiment (Type-0 learning) [49] which demonstrated that the (innate) architecture introduced here can account for 3.5 to 4.5-month-old infants' capability of displaying "object performance" behaviors in the draw-bridge experiment conducted by Rene Baillargeon [4], the application of the architecture discussed here for auditory and visual integrated learning for recognizing rotating objects (Type-2 multimodal learning) [55] and development of the value subsystem of the introduced architecture (Type-2, Type-1, and Type-0 learning) [10].

\section{Conclusions}

This is the first over-arching mental architecture that deals with autonomous development for every major module, the attention selector $T$ (early processing), the regressor (later processing), motor mapping (motor cortex), and the motivational system. Although the major modules of the architecture, such as the sensory mapping (T) [51] and the regressor [12,44], are not presented here, these modules and their automatic development have been studied and simulated.

The observation-driven MDP (Type-1) is more suited for autonomous mental development than the traditional MDP. This paper provides Type-1-Type-5 (DOSASE MDP) architectures, up to Type-6 (multilevel DOSASE MDP). A single DOSASE MDP can perform nonassociative learning, classical conditioning, instrumental conditioning, and general purpose reasoning and planning.

The proposed series of mental architectures might be useful for further understanding neuroscience and behavioral evidence and may inform more detailed models of cognitive development.

\section{Acknowledgments}

The work is supported in part by the National Science Foundation under Grant No. IIS 9815191, DARPA ETO under contract No. DAAN02-98-C-4025, DARPA ITO under Grant No. DABT63-99-1-0014, an MSU Strategic Partnership Grant, and research gifts from Microsoft Research and Zyvex. Many thanks to M. Badgero, Y. Chen, D. Cherba, C. Evans, J.D. Han, W.S. Hwang, X. Huang, K.Y. Tham, S.Q. Zeng, N. Zhang and Y. Zhang for their contributions to the SAIL and Dav projects as cited.
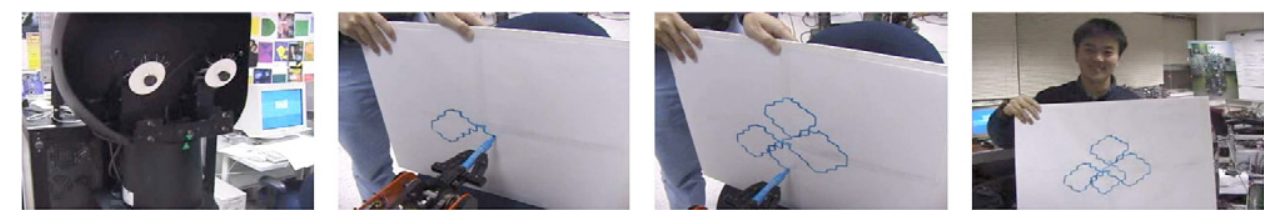

Fig. 13. The SAIL robot learned longer and more complex composite skills through transfer-and-chaining based on previously learned simpler and shorter skills, while it was interacting with human trainers in real-time. 


\section{References}

[1] J.S. Albus, Outline for a theory of intelligence, IEEE Trans. Syst. Man Cybern. 21 (3) (1991) 473-509.

[2] N. Almassy, G.M. Edelman, O. Sporns, Behavioral constraints in the development of neural properties: a cortical model embedded in a real-world device, Cereb. Cortex 8 (4) (1998) 346-361.

[3] J.R. Anderson, Rules of the Mind, Lawrence Erlbaum, Mahwah, NJ, 1993.

[4] R. Baillargeon, Object permanance in 3.5 and 4.5-month-old infants, Dev. Psychol. 23 (1987) 655-664.

[5] L. Birnbaum, M. Brand, P. Cooper, Looking for trouble: using causal semantics to direct focus of attention, in: Proceedings of the Fourth International Conference on Computer Vision, 1993, pp. $49-56$.

[6] R.A. Brooks, A robust layered control system for a mobile robot, IEEE J. Robot. Autom. 2 (1) (1986) 14-23.

[7] D.R. Cox, Statistical analysis of time series: some recent developments, Scand. J. Statist. 8 (2) (1981) 93-115.

[8] J.L. Elman, Learning and development in neural networks: The importance of starting small, Cognition 48 (1) (1993) 71-99.

[9] J.A. Feldman, D.H. Ballard, Connectionist models and their properties, Cognitive Sci. 6 (3) (1982) 205-254.

[10] X. Huang, J. Weng, Novelty and reinforcement learning in the value system of developmental robots, in: Proceedings of the Second International Workshop on Epigenetic Robotics (EPIROB'02), Edinburgh, Scotland, August 10-11, 2002, pp. 47-55.

[11] X. Huang, J. Weng, Value system development for a robot, in: Proceedings of the 2004 International Joint Conference on Neural Networks, July 26-29, 2004.

[12] W.S. Hwang, J. Weng, Hierarchical discriminant regression, IEEE Trans. Pattern Anal. Mach. Intell. 22 (11) (2000) 1277-1293.

[13] P.N. Johnson-Laird, Human and Machine Thinking, Lawrence Erlbaum, Hillsdale, NJ, 1993.

[14] L.P. Kaelbling, M.L. Littman, A.W. Moore, Reinforcement learning: a survey, J. Artif. Intell. Res. 4 (1996) 237-285.

[15] E.R. Kandel, J.H. Schwartz, T.M. Jessell (Eds.), Principles of Neural Science, fourth Ed., McGraw-Hill, New York, 2000.

[16] B. Kolb, I.Q. Whishaw, Fundamentals of Human Neuropsychology, third Ed., Freeman, New York, 1990.

[17] J.E. Laird, A. Newell, P.S. Rosenbloom, Soar: An architecture for general intelligence, Artif. Intell. 33 (1987) 1-64.

[18] A.R. Luria, The Working Brain, Penguin, Harmondsworth, England, 1973.

[19] D.W. Massoro, M.M. Cohen, L.A. Thompson, Visible language in speech perception: Lipreading and reading, Visible Language 22 (1) (1988) 8-31.

[20] D.E. Meyer, D.E. Kieras, A computational theory of executive cognitive processes and multiple-task performance: Part 1 basic mechanisms, Psychol. Rev. 104 (1997) 2-65.

[21] D.E. Meyer, D.E. Kieras, A computational theory of executive cognitive processes and multiple-task performance: Part 2 accounts of psychological refractory-period phenomena, Psychol. Rev. 104 (1997) 749-791.

[22] M. Minsky, Private Communication, 1999.

[23] U. Neisser, Cognitive Psychology, Appleton-Century-Crofts, New York, 1967.

[24] B.A. Olshansen, C.H. Anderson, D.C. Van Essen, A neurobiological model of visual attention and invariant pattern recognition based on dynamic routing of information, J. Neurosci. 13 (11) (1993) 4700-4719.

[25] D.N. Pandya, B. Seltzer, Association areas of the cerebral cortex, Trends Neurosci. 5 (1982) 386-390.

[26] V. Pratt, Thinking Machines, Basil Blackwell, Oxford UK, 1987.

[27] M.L. Puterman, Markov Decision Processes, Wiley, New York, 1994.

[28] L.R. Rabiner, A tutorial on hidden Markov models and selected applications in speech recognition, Proc. IEEE 77 (2) (1989) 257-286.
[29] D. Reisfeld, H. Woldson, Y. Yeshurun, Context-free attentional operators: the generalized symmetry transform, Int. J. Comput. Vision 14 (1995) 119-130.

[30] J.E. Richards (Ed.), Cognitive Neuroscience of Attention: A Developmental Perspective, Lawrence Erlbaum, Mahwah, New Jersey, 1998.

[31] A.J. Rudnicky, A.G. Hauptmann, K.F. Lee, Survey of current speech technology, Commun. ACM 37 (3) (1994) 52-57.

[32] L.R. Squire, E.R. Kandel, Memory: From Mind to Molecules, Scientific American Library, New York, 1999.

[33] R.J. Sternberg (Ed.), Thinking and Problem Solving, Academic Press, San Diego, California, 1994 (Chapters 1 and 2).

[34] R.S. Sutton, A. Barto, Reinforcement Learning, MIT Press, Cambridge, MA, 1998.

[35] J.K. Tsotsos, A 'complexity level' analysis of immediate vision, Int. J. Comput. Vision 1 (4) (1988) 303-320.

[36] J.K. Tsotsos, Analyzing vision at the complexity level, Behav. Brain Sci. 13 (1990) 423-469.

[37] A.M. Turing, Computing machinery and intelligence, Mind 59 (1950) 433-460.

[38] A. Van der Heijden, Selective Attention in Vision, Routledge, New York, 1992.

[39] L.S. Vygotsky, Thought and Language, MIT Press, Cambridge, MA, 1962 trans. E. Hanfmann, G. Vakar.

[40] A. Waibel, K. Lee, Readings in Speech Recognition, Morgan Kaufmann, San Mateo, CA, 1990.

[41] C. Watkins, P. Dayan, Q-learning, Mach. Learn. 8 (1992) 279-292.

[42] J. Weng, A theory for mentally developing robots, in: Proceedings of the IEEE Second International Conference on Development and Learning (ICDL 2002), June 12-15, MIT, Cambridge, MA, 2002, pp. 131-140.

[43] J. Weng, Developmental robotics: Theory and experiments, Int. J. Humanoid Robot. 1 (2) (2004) 199-235.

[44] J. Weng, W. Hwang, Online image classification using IHDR, Int. J. Document Anal. Recognition 5 (2-3) (2002) 118-125.

[45] J. Weng, W.S. Hwang, An incremental learning algorithm with automatically derived discriminating features, in: Proceedings of the Asian Conference on Computer Vision, Taipei, Taiwan, January 8-9, 2000, pp. 426-431.

[46] J. Weng, W.S. Hwang, Y. Zhang, C. Evans, Developmental robots: Theory, method and experimental results, in: Proceedings of the Second International Conference on Humanoid Robots, Tokyo, Japan, October 8-9, IEEE Press, 1999, pp. 57-64.

[47] J. Weng, J. McClelland, A. Pentland, O. Sporns, I. Stockman, M. Sur, E. Thelen, Autonomous mental development by robots and animals, Science 291 (5504) (2001) 599-600.

[48] J. Weng, I. Stockman, Autonomous mental development: Workshop on development and learning, Artif. Intell. Magazine 23 (2) (2002) 95-98.

[49] J. Weng, Y. Zhang, Y. Chen, Developing early senses about the world: 'object permanence' and visuoauditory real-time learning, in: Proceedings of the International Joint Conference on Neural Networks, Portland, Oregon, July 20-24, 2003.

[50] D.J. Wood, J.S. Bruner, G. Ross, The role of tutoring in problemsolving, J. Child Psychol. Psychiatry (1976) 89-100.

[51] N. Zhang, J. Weng, A developing sensory mapping for robots, in: Proceedings of the IEEE Second International Conference on Development and Learning (ICDL 2002), June 12-15, MIT, Cambridge, MA, 2002, pp. 13-20.

[52] N. Zhang, J. Weng, X. Huang, Progress in outdoor navigation by the SAIL developmental robot, in: Proceedings of the SPIE International Symposium on Intelligent Systems and Advanced Manufacturing, vol. 4573, October 28-November 2, Newton, MA, 2001.

[53] Y. Zhang, J. Weng, Grounded auditory development by a developmental robot, in: Proceedings of the INNS-IEEE International Joint Conference on Neural Networks, Washington, DC, July 14-19, 2001, pp. 1059-1064. 
[54] Y. Zhang, J. Weng, Action chaining by a developmental robot with a value system, in: Proceedings of the IEEE Second International Conference on Development and Learning (ICDL 2002), June 12-15, MIT, Cambridge, MA, 2002, pp. 53-60.

[55] Y. Zhang, J. Weng, Conjunctive visual and auditory development via real-time dialogue, in: Proceedings of the Third International Workshop on Epigenetic Robotics (EPIROB'03), Boston, MA, August 4-5, 2003, pp. 974-980.

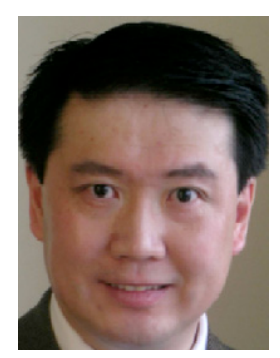

Juyang (John) Weng received his Ph.D. degree in Computer Science from University of Illinois, Urbana, IL USA, January 1989. His research interests include mental architectures, computational neuroscience, mental development, biologically inspired neural systems, vision, audition, touch, human-machine multimodal interface, and intelligent robots. $\mathrm{He}$ is the author or coauthor of over 180 reviewed research articles and book chapters published in books, journals, conferences and workshops. He is an editor-in-chief of International Journal of Humanoid Robotics and the founding Chairman of the Governing Board of the multidisciplinary International Conferences on Development and Learning (ICDL) (http://cogsci.ucsd.edu/ triesch/icdl/). He was the founding chairman of the Autonomous Mental Development Technical Committee of the IEEE Computational Intelligence Society (2004-2005), an associate editor of the IEEE Trans. on Pattern Recognition and Machine Intelligence, an associate editor of the IEEE Trans. on Image Processing, a program chairman of the NSF/DARPA Workshop on Development and Learning (WDL), held April 2000 at Michigan State University (MSU), East Lansing, MI (http://www.cse.msu.edu/dl/), and a program chairman of the 2nd International Conference on Development and Learning (ICDL02), held at Massachusetts Institute of Technology, Cambridge, MA, June 2002 (http://www.egr.msu.edu/icd102/). He initiated and supervised the SAIL (Self-organizing Autonomous Incremental Learner) and Dav projects, in which he and his coworkers have designed and custom built their SAIL and Dav robots for research on robotic computational realization of autonomous mental development. More detail is available on line at http://www.cse.msu.edu/weng/. 